\title{
3D discrete dislocation dynamic investigations of persistent slip band formation in FCC metals under cyclical deformation
}

\author{
Fanshi MENG ${ }^{\mathrm{a}}$, Emilie FERRIE ${ }^{\mathrm{a}}$, Christophe DEPRES ${ }^{\mathrm{b}}$, Marc FIVEL $^{\mathrm{a}, *}$ \\ ${ }^{a}$ Univ. Grenoble Alpes, CNRS, Grenoble INP, SIMaP, 38000 Grenoble, France \\ ${ }^{b}$ Laboratory SYMME, Université de Savoie, BP80439,74944 Annecy-le-Vieux Cedex, France
}

\begin{abstract}
The formation of persistent slip bands (PSBs) at early stages of fatigue plays an important role in fatigue damage. In order to get a better understanding of the physics involved at the dislocation scale, 3D discrete dislocation dynamic simulations are set up in the case of an isolated surface grain of $\mathrm{Cu}$ cyclically loaded in single slip. Simulations reveal the complex transformation process of the dislocation microstructure from a homogeneous distribution into an organized microstructure made of slip bands mainly composed of dislocation dipoles and prismatic loops. These slip bands are the embryo of the PSBs observed for large number of cycles. The localization mechanism evidenced by the simulations is well explained from a stress field analysis which highlights the role of cross-slip. A saturation regime is reached after a few cycles during which dislocation microstructure, dislocation densities and height of extrusions/intrusions remain unchanged. Furthermore, the linear relationship between the volume fraction of slip bands and the imposed plastic strain amplitude is retrieved by the simulations and the stability of the slip bands is verified by decreasing the plastic strain amplitude after the saturation regime is reached. Finally, when compared to AISI 316L stainless steel, $\mathrm{Cu}$ shows a tendency to build more bands with relatively larger thicknesses which is attributed to the differences in the cross-slip probability.
\end{abstract}

Keywords: Discrete dislocation dynamics, Fatigue simulation, Cu single crystal, Persistent slip band, Cyclic irreversibility

2010 MSC: 00-01, 99-00

\section{Introduction}

Fatigue damage is one of the main origin of structure failure in long terms, especially for aerospace and power plant industry [1, 2. For example, in nuclear industry, materials at the mixing zone of hot fluid and cold fluid in the piping system of nuclear plant always endure thermal fatigue shocks due to the temperature 5 gradients, which may ultimately lead to leakage of cooling water (Accident Civaux [3]). Simular failure problems are observed in airplane components or in any structural materials subjected to repetitive loads 44. Investigations of the fatigue damage mechanism is thus a major concern for industry and academic research. Since the first systematic fatigue research conducted by August Wöhler [5] on railway axles, fatigue damage mechanism has been investigated for more than 100 years. However, due to the complexity of the underlying physics in terms of time and space scales, a complete understanding of the fatigue failure mechanism is still not available. The fatigue damage process is generally divided into two stages [6]: the initiation and the propagation of cracks. The initiation stage represents about $90 \%$ of the fatigue life, which is also called the early stage of fatigue damage. The work presented here exclusively focuses on the early stage of fatigue damage.

\footnotetext{
Fully documented templates are available in the elsarticle package on CTAN

* Corresponding author

Email address: Marc.Fivel@grenoble-inp.fr (Marc FIVEL)
} 
One remarkable observation during experiments of the early stages of fatigue damage is the formation of slip bands, which was firstly evidenced by Ewing [7] at the surface of a Swedish iron. During the last fifty years, the fatigue behavior of FCC (face-centered cubic) metals has been deeply investigated [8, 9, 10] because of the relatively uniformed fatigue behaviors and wide applications in industry. The development of persistent slip bands (PSBs) [8, 11, 12, 13 is considered as a characteristic feature for FCC metals at early stages of fatigue damage. Different aspects concerning PSBs have been studied extensively such as the dislocation microstructures within the PSBs [4, 8, the surface roughness evolution at the PSB's surface [14, 15] and the dislocation motion mechanism [16. The recent publication of Lavenstein et al. [17] has reported dynamic observations of the formation of PSBs at a micro-scale. In situ SEM observations of Ni microcrystals oriented for single slip showed that the PSBs nucleate from inside the crystal and gradually grow in the sample until they reach the free surfaces. It was also concluded that the location of the PSBs corresponds to the positions of the slip traces evidenced during the first 10 cycles.

Among the FCC metals, $\mathrm{Cu}$ single crystal is the most studied material and commonly considered as a reference for academic study of FCC crystals. The evolution of the dislocation microstructure with the applied strain amplitude of $\mathrm{Cu}$ in single slip situation has been well explained by Mughrabi [8] by introducing

30 his famous CSS (cyclic stress-strain) curve: for small plastic strain amplitude, a matrix and vein structure is formed; for larger strain amplitudes, PSBs are formed; for even larger applied deformation, the activation of a second slip system [18] leads to the formation of dislocation cells or labyrinth structures. The formation of PSBs is always accompanied by a saturation phenomenon [8, 4] regarding both the macroscopic response and the PSBs volume fractions and positions.

On the other hand, fatigue behavior is affected by many factors. Since the applied plastic strain amplitude influences the dislocation behaviors, it has a strong effect on the PSB volume fraction [12, the hardening rate [19] and the shape of the macroscopic response [20. Winter [12 has proposed a model relating the volume fraction of PSB with the applied strain amplitude through a linear relationship. Even though the fatigue behaviors of most of the FCC metals are quite similar, slight differences exist between them. By

40 comparing copper-aluminum alloy with pure $\mathrm{Cu}$, Feltner and Laird 21] found different sensitivities to the initial conditions, which allows the authors to bring out the influence of the stacking fault energy (SFE) on the cyclic slip mode [22. Later on, short-range order (SRO) 23, 24] in alloys and annihilation distance $y_{s}$ 25. were also pointed out to characterize the slip mode. Despite these studies, no unified criterion of the slip mode transition has been proposed yet.

It can be seen that dislocation motion in fatigue damage is crucial but always difficult to be traced with experimental methods. On the other hand, numerical simulations offer new possibilities. The recent 3D Discrete Dislocation Dynamics (DDD) model is a mesoscopic simulation method which aims at relating the dislocation activity to the mechanical response of micrometric size volumes. Proposed in the early 1990s [26, 27, DDD possesses special advantages in studying the collective behavior of massive dislocation

50 populations because most of the physics of mechanisms for dislocations are taken into account, eventually complemented by reasonable constitutive rules or empirical laws when continuum elastic framework is not sufficient (i.e. dislocation nucleation, grain boundary interaction, etc..). When both in situ and postmortem experiments can only provide limited information, DDD shed new light on the understanding of fatigue damage at the mesoscopic scale.

Considering the computer capacity, the application of DDD to fatigue started with 2D simulations. In a very simplified 2D DDD model, Yang et al. 28] have observed that randomly distributed dislocations could evolve to form a regular matrix-wall structure in $\mathrm{Cu}$. Deshpande et al. [29] studied the interaction between dislocations and pre-existing cracks by 2D DDD. Brinckmann and Van der Giessen [30] also used 2D DDD to investigate the stress concentration during the early fatigue cycles. In three dimensions, the most extensive DDD investigations of fatigue were conducted by Déprés et al. [31, 32] (see also [33] for a review of this work) who used a 3D DDD edge-screw model to study the Low Cycle fatigue behavior of AISI 316L stainless steel loaded in a single slip mode. The main result of this study is the prediction of the number of cycles required to initiate a crack and its dependence on material parameters. More recently, they have investigated the propagation of fatigue cracks inside a grain [34] and into the neighbor grains [35].

Later on, more attention was paid to multiple slip like Hussein and El-Awady who used a nodal DDD model to investigate the microstructure characters developed in a fatigued Ni grain in multiple-slip situation 
[36] and the surface roughness of Ni single crystals [37. They pointed out specifically the influence of the initial condition and grain size on the macroscopic response and localization of plasticity at the surface. El Achkar and Weygand [38] recently used graph analysis to quantify the evolution of the dislocation 70 microstructure in terms of prismatic loops and dipoles developed in a surface grain of Al in multiple slip fatigue loading conditions. Erel et al. 39 have artificially introduced a wall structure which mimics the microstructure configuration of a typical PSB observed experimentally in 3D DDD in order to study the movement of dislocations within the wall and the relationship with cyclic hardening and saturation stress. Several researchers have also applied DDD to study the irreversibility under cyclic torsion loading [40, 41, 75 as well as the Bauschinger effect in thin films [42, 43.

Surprisingly, although DDD has already been applied to study the fatigue behavior of FCC metals, the cyclical mechanical behavior of $\mathrm{Cu}$ during single slip has never been studied in details despite the fact that $\mathrm{Cu}$ is certainly the most studied metal in classic experimental fatigue research and well documented. Moreover, the localization mechanism associated to the formation of multiple persistent slip bands has never

${ }_{80}$ been simulated. Finally, to the best of the authors' knowledge, no DDD simulation of the saturation regime observed experimentally has been reported in the literature. Therefore, in this article, we shall use DDD model to simulate the fatigue behavior of $\mathrm{Cu}$ in single slip mode with the aim of elucidating the formation and propagation mechanisms at the origin of the PSBs. The paper is written as follows. Next section is a brief presentation of the 3D DDD model and its adaptation to treat fatigue simulations of a surface grain.

85 Then simulations results are exposed in the next section, starting with a careful examination of the PSB formation and multiplication mechanisms, followed by a one to one comparison between $\mathrm{Cu}$ and ASI316L stainless steels behaviors.

\section{Simulation methods}

\subsection{Screw-edge model}

The DDD model retained for this study is the edge-screw model TRIDIS [27] previously used in the modeling of Low Cycle Fatigue of AISI 316L stainless steels [33. In this model, dislocation lines are divided into connected discrete segments of edge and screw character. The velocity of the dislocation segments is calculated by a linear mobility law with the effective shear stress $\tau_{e f f}$, resolved on the dislocation slip system:

$$
v=\frac{\tau_{e f f} * b}{B}
$$

where $v$ is the velocity of the dislocation segment, $b$ is the length of the Burgers vector and $B$ is a phonon drag coefficient. $\tau_{\text {eff }}$ is a combination of different stress fields acting on the dislocation segment:

$$
\begin{gathered}
\tau_{e f f}=\tau^{\prime}-\operatorname{sign}\left(\tau^{\prime}\right) * \tau_{f r} \\
\tau^{\prime}=\left\{\left[\frac{1}{b}\left(\boldsymbol{\sigma}_{a p p}+\boldsymbol{\sigma}_{i n t}\right) \boldsymbol{b}\right] \times \boldsymbol{l}\right\} * \boldsymbol{g}+\tau_{l t}
\end{gathered}
$$

In these two equations, $\boldsymbol{b}$ is the Burgers vector, $\boldsymbol{l}$ is the line vector of the segment and $\boldsymbol{g}$ is the glide direction vector contained in the glide plane and perpendicular to the dislocation line. The shear stress $\tau^{\prime}$ is the stress resolved on the dislocation segment slip system obtained from the superposition of different stress fields: $\boldsymbol{\sigma}_{a p p}$, the applied stress field; $\boldsymbol{\sigma}_{i n t}$, the internal stress field produced by the other dislocations contained in the crystal; $\tau_{l t}$ is the line tension correction. Finally, the effective shear stress $\tau_{\text {eff }}$ is obtained by subtracting the contribution of the lattice friction stress $\tau_{f r}$, also called the Peierls stress which refers to the impediment caused by the crystal lattice on the dislocation movements. Note that in the present simulations we will neglect the image stress field that shall arise from the interaction between dislocations and the grain free surface since 34 has shown that they could be neglected in fatigue simulation of surface grains.

When the effective shear stress $\tau_{\text {eff }}$ is bigger than the friction stress $\tau_{f r}$, the dislocation $i$ is able to move. Then the predicted displacement $\Delta d^{i}$ is estimated in an explicit way by multiplying the time step $\delta t$ 
and velocity $v^{i}: \Delta d^{i}=v^{i} * \delta t$. In practice, the actual distance swept by the dislocation segment may be reduced by the presence of obstacles within the flight area. The associated shear strain is then calculated as: $\Delta \gamma_{p}^{i}=b * \frac{\Delta A^{i}}{V}$, where $V$ is the volume of studied grain, $\Delta A^{i}=l^{i} * \Delta d^{i}, \Delta A^{i}$ is the area swept by the dislocation segment $i$ and $l^{i}$ is the length of the segment $i$. The increment of the plastic strain, $\Delta \varepsilon_{p}$ accumulated in the simulated grain is defined as the sum of the individual contributions $\Delta \varepsilon_{p}^{i} \operatorname{coming}$ from all the dislocation segments and calculated as:

$$
\begin{gathered}
\Delta \varepsilon_{p}^{i}=\frac{1}{2 b}\left(\boldsymbol{b}^{(s)} \otimes \boldsymbol{n}^{(s)}+\boldsymbol{n}^{(s)} \otimes \boldsymbol{b}^{(s)}\right) \Delta \gamma_{p}^{i} \\
\Delta \varepsilon_{p}=\sum_{i=1}^{N} \Delta \varepsilon_{p}^{i}
\end{gathered}
$$

where $s$ is the slip system of the dislocation segment and $\boldsymbol{n}^{s}$ and $\boldsymbol{b}^{s}$ are the slip plane normal and Burgers vector of this slip system, respectively. An equivalent Von Mises plastic strain is calculated from the second invariant of the plastic strain tensor as:

$$
\varepsilon_{p}^{V M}=\frac{2}{3} \sqrt{\frac{3\left(\varepsilon_{x x}^{2}+\varepsilon_{y y}^{2}+\varepsilon_{z z}^{2}\right)}{2}+\frac{3\left(\varepsilon_{x y}^{2}+\varepsilon_{x z}^{2}+\varepsilon_{y z}^{2}\right)}{4}}
$$

where $\varepsilon_{i j}$ are the components of the plastic strain tensor.

For each time step of the calculation $\delta t$, the following interactions are taken into consideration: annihilation and combinations of dislocations, junction formations, cross-slips and line reconnections. In TRIDIS [27, the cross-slip probability for the screw segments is computed from the following expression adapted from 44 :

$$
P_{i}=\beta \frac{l}{l_{0}} \frac{\delta t}{t_{0}} \exp \left(\frac{\tau_{d e v}-\tau_{I I I}}{k T} V_{a c t}\right)
$$

where $\beta=10^{5}$ is a normalization coefficient to assure that the cross-slip probability $P_{i} \in[0,1], \frac{l}{l_{0}}$ is the ratio between the length of the considered screw dislocation segment $l$ and the reference length $l_{0}\left(l_{0}=1 \mu \mathrm{m}\right.$ [27] and $\frac{\delta t}{t_{0}}$ is the ration between the simulation time step $\delta t$ and the reference time step $t_{0}\left(t_{0}=1 s[27]\right)$. Inside the exponential function, the entire formula represents the activation energy for cross-slip: $\left(\tau_{d e v}-\tau_{\text {III }}\right)$ is the omparison between the absolute value of the shear stress resolved on the deviate plane and in the direction of the Burgers vector of the dislocation segment, $\tau_{d e v}$ and a reference stress value, $\tau_{I I I}$ corresponding to the shear stress measured at the beginning of Stage III in single crystals tensile tests; $V_{a c t}$ is the activation volume, $k$ and $T$ represent the Boltzman constant and the temperature respectively.

In practice, cross slip is decided for screw dislocation segments if the following checks are successively verified :

- the screw segment is part of a dislocation line admitting an angle with the Burgers vector smaller than 10 degrees,

- the shear stress resolved in the deviate slip system is larger than the shear stress resolved on the primary slip system $\tau_{\text {dev }} / \tau>1.15$.

- a random number generated by the computer is lower than the theoretical probability $P i$.

This algorithm implies that cross-slip mechanism in DDD is a stochastic event.

\subsection{Quasi-static single shear loading}

The applied stress field $\boldsymbol{\sigma}_{a p p}$ is imposed as a homogeneous stress tensor, identical for each dislocation segment. The applied stress field at step $k$ is given as follows:

$$
\boldsymbol{\sigma}_{a p p}^{k}=\boldsymbol{\sigma}_{a p p}^{k-1}+\lambda *(-1)^{a} * \Delta \boldsymbol{\sigma}_{a p p}
$$


where $\boldsymbol{\sigma}_{a p p}^{0}=\mathbf{0}, a=0$ for loading so that $(-1)^{a}=1$, and $a=1$ for unloading so that $(-1)^{a}=-1$. $\lambda$ is the coefficient to control the quasi-static loading rate. $\Delta \boldsymbol{\sigma}_{a p p}$ is the stress increment at each time step of the proportional applied loading. The stress tensor is chosen so that it induces a pure shear stress on $\frac{a}{2}(1 \overline{1} \overline{1})[110]$ slip system. When expressed in the crystal coordinate system, it writes as:

$$
\Delta \boldsymbol{\sigma}_{\text {app }}=\left(\begin{array}{ccc}
0.577 & 0 . & -0.288 \\
0 . & -0.577 & -0.288 \\
-0.288 & -0.288 & 0 .
\end{array}\right) * \dot{\sigma} * \delta t
$$

In $\mathrm{Eq} 9, \dot{\sigma}$ is the stress rate $\left(\dot{\sigma}=5 \times 10^{9} \mathrm{MPa} / \mathrm{s}\right)$. Let recall here that the time integration algorithm is purely explicit. For this reason, the choice of the time step $\delta t$ is of great importance. If $\delta t$ is too small, the computational time can be very long. On the other hand, if $\delta t$ is too big, the local interaction cannot be well simulated. According to validation tests, $\delta t$ is chosen as $\delta t=2 \times 10^{-10} \mathrm{~s}$.

$\lambda$ at step $k$ is computed from the increment of the equivalent plastic strain at step $k-1, \Delta \varepsilon_{p}^{V M, k-1}$. When $\Delta \varepsilon_{p}^{V M, k-1}$ is smaller than the value $\Delta \varepsilon_{p}^{V M, \min }$, the dislocations are considered in an equilibrium position so that the amplitude of the applied stress should be increased and $\lambda=1$. In the ideal situation, $\Delta \varepsilon_{p}^{V M, \min }$ should be zero, but small vibration of dislocations can never be avoided. The choice $\Delta \varepsilon_{p}^{V M, \text { min }}=10^{-7}$ has been decided in an empirical manner by carefully monitoring the dislocation activity during the simulations. If $\Delta \varepsilon_{p}^{V M, k-1}$ is bigger than the value $\Delta \varepsilon_{p}^{V M, \max }$, the increment of deformation at last step is judged to be too high and may lead to dislocation avalanches which violates the assumption of quasy-static loading conditions. In this situation, it is deciced that the applied stress is reduced by choosing $\lambda=-0.5$. Monitoring of the dislocation dynamics during cycling has lead to $\Delta \varepsilon_{p}^{V M, \max }=10^{-5}$. If the increment of deformation $\Delta \varepsilon_{p}^{V M, k-1}$ is between the two values, the applied stress remains unchanged and $\lambda=0$ so that the dislocations can keep on moving under the same applied stress field to find the equilibrium position. The value of $\lambda$ at step $k$ is summarized as follows:

$$
\lambda=\left\{\begin{array}{lll}
1 & \text { if } & \Delta \varepsilon_{p}^{V M, k-1} \leq \Delta \varepsilon_{p}^{V M, \min } \\
-0.5 & \text { if } & \Delta \varepsilon_{p}^{V M, k-1} \geq \Delta \varepsilon_{p}^{V M, \max } \\
0 & \text { else } &
\end{array}\right.
$$

The load is reversed when the equivalent accumulated plastic strain reaches the desired maximum imposed strain amplitude. In the next simulations, the load is always symmetric $(R=-1)$ so that an imposed plastic strain amplitude $\varepsilon_{p, \text { applied }}^{V M}=10^{-3}$ signifies that the plastic strain will vary between $-10^{-3}$ and $125+10^{-3}$.

\subsection{Simulation settings}

The grain geometry and the initial dislocation configuration are selected so that it ensures strain localization on a particular slip system and simulations could be performed in a reasonable CPU time. These informations are provided by the previous work of Déprés et al [31] who demonstrated two key features.

130 Firstly, strain localization is possible on slip systems admitting a low angle between the Burgers vector and the normal to the free surface. Secondly, the PSB microstructure can be obtained from a single initial Frank-Read source, i.e. without the need to include all the 12 possible slip systems.

In our case, the simulated situation is a [221] surface grain as shown in Fig 1. For sake of simplicity the grain geometry is defined as an opened cylinder of $D=5 \mu \mathrm{m}$ diameter and $H=5 \mu m$ height. Its top surface (as shown in yellow in Fig 1) is considered as a free surface where dislocations can escape and leave the crystal, while the other surfaces are all impenetrable for the passage of dislocations. The resolution of the discrete lattice of the edge-screw model is fixed to $\mathrm{xl}=10 \mathrm{~b}$, where $b$ is the Burgers vector [? ]. A single Frank-Read dislocation source is introduced inside the crystal on the $\frac{a}{2}(1 \overline{1} \overline{1})[110]$ slip system, refered as the primary slip system. The applied stress field defined by Eq9 induces a pure shear stress of the primary slip system. Although DDD simulations will only concern two families of dislocations, namely $\frac{a}{2}\left(\begin{array}{lll}1 & \overline{1} & \overline{1}\end{array}\right)\left[\begin{array}{lll}1 & 1 & 0\end{array}\right]$ and $\frac{a}{2}(1 \overline{1} 1)[110]$, the ratio between the shear stress for each of the total 12 systems and the shear stress on the primary slip system $\frac{\tau^{\text {system }}}{\tau^{\text {primary }}}$ are given in Tab 1 for information. 
The green plane drawn in Fig 1 is a typical slip plane of the primary slip system \#8. The blue plane is a slip trace of system \#7 which corresponds to the conjugate slip plane of the primary system. System \#7 will be later called the deviate slip plane since dislocation can cross-slip from system \#8 to system \#7 during the fatigue simulations.

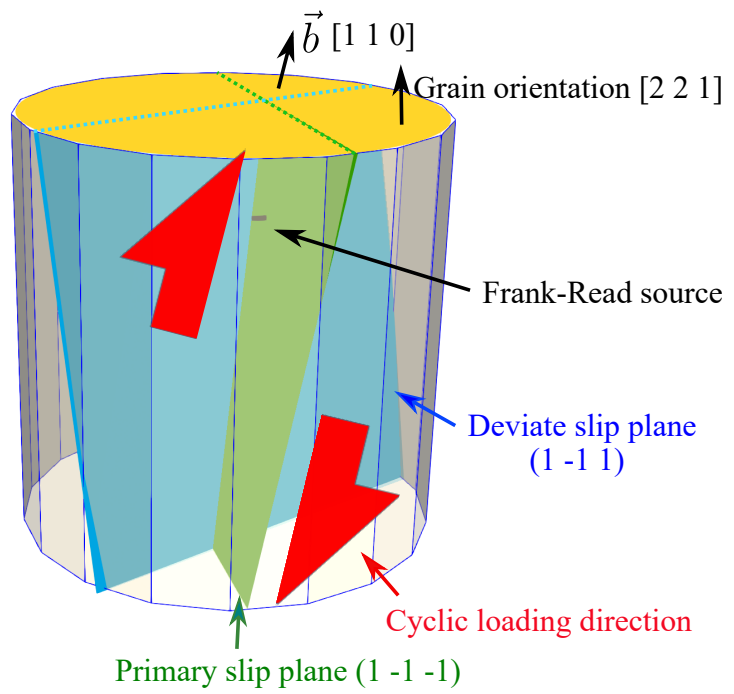

Figure 1: Illustration of the grain shape, the relative positions of the slip systems, the initial condition, and the loading mode used in the DDD simulations.

Table 1: Slip systems in FCC crystals and activation factor induced on each system by the stress tensor given in Eq9 Primary slip system corresponds to slip system \#8 and deviate slip system is system \#7.

\begin{tabular}{lcccccccccccc}
\hline Slip system & 1 & 2 & 3 & 4 & 5 & 6 & $\underline{\mathbf{7}}$ & $\underline{\mathbf{8}}$ & 9 & 10 & 11 & 12 \\
\hline Burgers vector & {$[\overline{1} 01]$} & {$[\overline{1} 01]$} & {$[011]$} & {$[011]$} & {$[\overline{1} 10]$} & {$[\overline{1} 10]$} & {$[110]$} & {$[110]$} & {$[01 \overline{1}]$} & {$[01 \overline{1}]$} & {$[101]$} & {$[101]$} \\
Slip plane & $(111)$ & $(1 \overline{1} 1)$ & $(\overline{1} \overline{1} 1)$ & $(1 \overline{1} 1)$ & $(111)$ & $(11 \overline{1})$ & $(1 \overline{1} 1)$ & $(1 \overline{1} \overline{1})$ & $(111)$ & $(\overline{1} 11)$ & $(11 \overline{1})$ & $(1 \overline{1} \overline{1})$ \\
$\frac{\tau^{\text {system }}}{\tau_{\text {primary }}}$ & 0.50 & 0.17 & 0.50 & 0.17 & 0.67 & 0.67 & 0.33 & 1.00 & 0.17 & 0.50 & 0.17 & 0.50 \\
\hline
\end{tabular}

Most of our simulations are realized for $\mathrm{Cu}$ but for sake of comparison, some complementary simulations are also performed for a second material having a higher activation volume, i.e. a lower stacking fault energy. In that objective, a comparison has been made between $\mathrm{Cu}$ and austenitic stainless steel AISI 316L. All the parameters used in the DDD simulations for these two materials are listed in Tab 2 and taken from [32, 27.

Table 2: Parameters used in the simulations for $\mathrm{Cu}$ and AISI 316L

\begin{tabular}{cccccccc}
\hline & Shear modulus & Poisson & Burgers vector & Friction & Drag coefficient & \\
stress & $\begin{array}{c}\text { Patio } \\
\text { Parameters }\end{array}$ & $\begin{array}{c}b \\
10^{-10} m\end{array}$ & $\begin{array}{c}\tau_{f r} / \mu \\
\text { Pa.s }\end{array}$ & $\begin{array}{c}\tau_{\text {act }} \\
\mathrm{MPa}\end{array}$ \\
& $\mathrm{MPa}$ & $\nu$ & 2.56 & $50 \times 10^{-5}$ & $1.5 \times 10^{-5}$ & $300 b^{3}$ & 30 \\
AISI 316L & 42000 & 0.324 & 2.54 & $50 \times 10^{-5}$ & $0.71 \times 10^{-5}$ & $1800 b^{3}$ & 56 \\
\hline
\end{tabular}

\subsection{Spatial dislocation distributions measurement}

Since the simulated situation is single slip, most of the dislocations are primary dislocations slipping on system \#8. For the analysis of Persistent Slip Bands formation, it is important to quantify the distribution 
of the dislocation positions with time. The proposed method is illustrated in Fig 2, Let assume that the two labeled planes (Plane 1 and Plane 2) in Fig 2 are two primary slip planes. The total length of dislocation lying on each slip plane, noted as $L 1$ and $L 2$, is calculated. The evolution of the total dislocation length on every slip plane of the grain is plotted with the relative plane position calculated along the direction of the normal to the slip plane (as shown in Fig 2). With this method, the spatial distribution of the primary dislocations along the transverse direction is calculated and the obtained histograms give information on the dislocation density distribution in the grain: peaks stand for slip bands. If needed, the quantitative evolution of positive or negative dislocations is a possible outcome of this method.

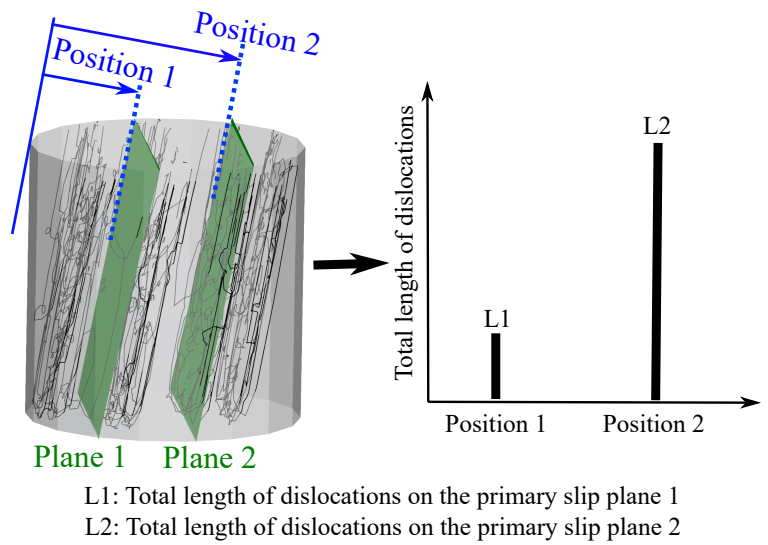

Figure 2: Illustration of the method used to quantify the dislocation distributions.

\section{Results and discussions}

\subsection{Formation process and distribution evolution of the slip bands}

This section presents the simulation of $\mathrm{Cu}$ in a cylindrical grain shown in Fig $1(D=H=5 \mu m)$ under $\varepsilon_{p, \text { applied }}^{V M}=10^{-3}$ applied plastic strain amplitude. The dislocation distributions computed at maximal deformation is shown in Fig 3(a) for different cycle numbers. After the first two cycles, multiple dislocation loops have been generated from the initial Frank-Read source in order to accommodate the imposed plastic strain. During this multiplication process, the dislocations are homogeneously distributed in the entire grain through the double cross-slip mechanism as also evidenced in [45]. As can be seen in Fig 3(a), the distribution remains homogeneous until the $5^{\text {th }}$ cycle. However, from the $10^{\text {th }}$ cycle, a localization of dislocations can be observed. Starting from the $10^{\text {th }}$ cycle, some dislocations gather to form adjoining slip planes connected by dislocations of the cross-slip system. The built-up microstructure compares well to the macro slip bands as observed in experiments 4, 46. Most of the plastic strain is confined in these bands. In this paper, the name PSB will be used to describe this organized microstructure although at this stage this dislocation organization obviously corresponds more to the embryo of the PSBs that will form later in the cycling. According to Lavenstein et al. [17, the slip traces observed during the first cycles coincide with the PSBs. These simulated bands can therefore be considered as the embryos of the PSBs.

The dislocation arrangements inside the PSBs are relatively much denser than everywhere else in the grain but randomly distributed dislocations are still visible. With the increase of the number of cycles, more PSBs are constructed (see Fig 3(a)). After the $15^{\text {th }}$ cycle, besides the two already formed PSBs, the embryo of the third one appears at the right side of the grain. From the $20^{\text {th }}$ cycle, four PSBs are about to be formed but the frontiers between the PSBs are still not very clear because of the existence of many reversible dislocations. After the $26^{\text {th }}$ cycle, four PSBs have been well constructed and easy to be distinguished directly from the $3 \mathrm{D}$ picture. The width of the formed PSB is about $1 \mu \mathrm{m}$, which is of the same order of magnitude as observed in the micro-sample fatigue experiment of Lavenstein et al. [17. Finally, it is found that the positions and thicknesses of these four PSBs stay almost invariant from the $26^{\text {th }}$ cycle to the $50^{\text {th }}$ cycle 
which could then be qualified as a saturation regime similar to what was observed experimentally in [4, 46]. It is worth mentioning also that the distribution process of the numerical PSBs presented here resembles to the in-situ SEM observations printed in Figure 1 of Lavenstein et al. 2020 [17].

(a)

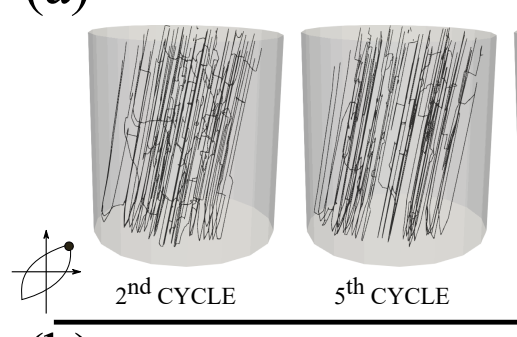

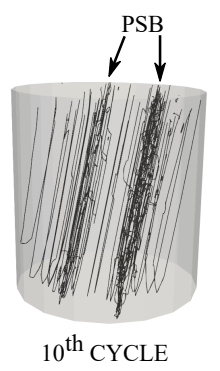

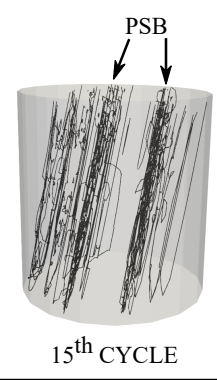

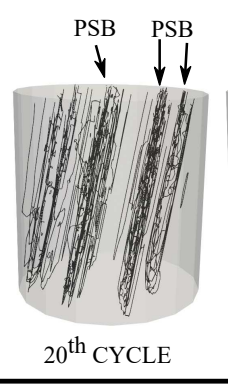

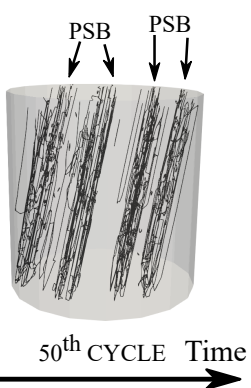

$26^{\text {th }}$ CYCLE $\quad 50^{\text {th }}$ CYCLE Time

(b) $\times 10^{13}$
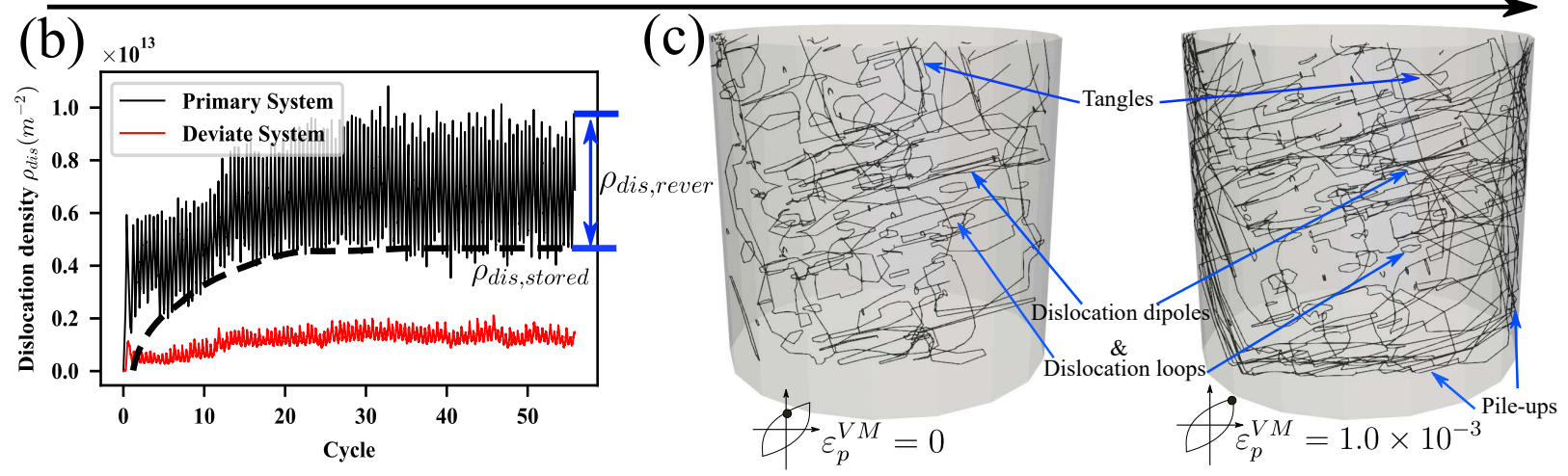

Figure 3: Simulation results for a cylindrical $\mathrm{Cu}$ grain $(D=H=5 \mu m)$ at $\varepsilon_{p \text {,applied }}^{V M}=10^{-3}$ : (a) Evolution of the dislocation microstructure with the increasing cycles at maximal deformation; (b) dislocation density evolution for primary and deviate slip systems with loading cycles; (c) snapshots of the dislocation microstructure inside a PSB formed at cycle 26 (see green box) observed at zero and maximal deformation.

In order to quantify the organization of the dislocation microstructure during the cyclic loading, the dislocation densities on the primary and cross-slip slip systems (or deviate slip system) are plotted as functions of the loading cycles in Fig 3(b). During one cycle loading, the dislocation densities for both slip systems fluctuate with the increasing or decreasing of the strain. Thus, the dislocation density reaches two extrema per cycle, one for the maximum positive strain and a second one for the minimum negative plastic strain. The plot shows that the dislocation population can be subdivided in two parts: $\rho_{\text {dis }}=$ $\rho_{\text {dis,stored }}+\rho_{\text {dis,rever. }}$. The stored dislocation $\rho_{\text {dis,stored }}$ bluecan be estimated as the fit of the minimum of the curve. It is plotted as a dashed line in Fig 3 (b). The evolution of the reversible dislocation $\rho_{\text {dis, rever }}$ corresponds to the fluctuations within each cycle.

The reversible dislocations mainly contribute to the accommodation of the plastic deformation during one cycle. The stored dislocations have lower mobility. They accumulate inside the crystal and contribute to the formation of the microstructures. The evolution of the stored dislocation densities matches well with the formation of the PSBs. When the four PSBs are still under construction (before cycle 26), the stored dislocation density is still increasing and the amplitude of the reversible dislocation density is quite low. Once the PSBs are all formed (after cycle 26), the stored dislocation density remains constant and the amplitude of the reversible dislocation density reaches a maximum. The microstructure constructed by a limited number of stored dislocations (at cycle 26) described hereafter.

The detailed structure of the dislocation arrangement within a PSB at the $26^{\text {th }}$ cycle (visualized in the green box in Fig 3(a)) is presented in Fig 3(c) when the VonMises equivalent plastic strain is zero and when it is maximal. Practically, Fig 3(c) is a slab of Fig 3(b)-cycle 26 viewed along direction (1111) so that the dislocations of the primary slip system are seen in their real length. When $\varepsilon_{p, a p p l i e d}^{V M}=0$, dislocations exist in the form of dipoles, prismatic loops and tangles. Multiple dislocation dipoles are built-up by the 
attraction of two edge dislocations of opposed sign and are arranged in a regular array. In the center of the PSB, due to the back and forth motion of dislocations by cross-slip during the loading and unloading sequences, dislocation dipoles are cut into pieces by line multiple recombinations which create dislocation prismatic loops. This cutting mechanism has already been described in [31, 47, 9]). Tangled dislocations are also formed from the recombination process on the side of the prismatic loops. The simulated dislocation microstructures are quite similar to the experimental results. The existence and persistence of dislocation dipoles under cyclic loading have been reported in several experimental studies [48, 49. When the applied plastic strain amplitude is increased, most of the dislocation lines or tangles are destroyed or extended by

220 the stronger applied shear stress at maximal deformation. In contrast, dislocation dipoles and prismatic loops show more permanence because most of them seldom move or are broken down by the stress. The low mobility of these structures suggests that they contribute little to the accommodation of the plastic deformation. Nevertheless, dislocation pile-ups are found to be built up near the grain boundaries at maximal deformation. Dislocation pile-ups have a relatively higher mobility since they can easily unstack

225 when the load is reversed. From this point, it can be inferred that during one cycle of deformation, most of the plastic deformation is accommodated by the pile-up dislocations. These observations also show that the build-up and build-down of the dislocation pile-ups correspond to the fluctuation of reversible dislocations while the persistence of dislocation dipoles and prismatic loops refers to the storage of dislocations.

In experiments, dislocation dipoles exist also in several different aggregation forms such as dipole bundles

230 [50], veins [51] and ladders [46] but the formation mechanism is still not clear and under investigation [48]. Due to the limitations of the model in term of computational power, the formation of the ladder and vein structure are not accessible by the DDD simulations but multipoles are clearly evidenced in the dislocation organization.

It is worth mentioning that the saturation state normally appears after 1000 cycles in experiments whereas in the DDD simulation, the saturation regime is observed after about 25 cycles. This difference suggests that the DDD simulation may accelerate the process of microstructure formation under cyclic loading. A possible reason could come from the physical mechanisms which are not accounted for in our DDD simulations such as forest hardening, point defect interactions, diffusion mechanism, dislocation/grain boundary interactions and the presence of the grain neighborhood. Note also that the relative small size of the simulated volume contributes to the accelerated organization of the dislocation microstructure. Nevertheless, the in-situ fatigue tests on micro-samples [17, have demonstrated that the strain localization that will ultimately lead to PSBs starts within the first 10 cycles. This statement can be considered as a favorable argument for the limited number of cycles reached in DDD simulation. Finally, despite these restrictions, it is remarkable that DDD did reproduce the main characteristics of dislocation self-organization in fatigue.

\section{3.2. Growth of the surface roughness at the surface}

Another important aspect of the early stages of fatigue is the growth of roughness on the surface at its intercept with the PSBs. In DDD, the plastic steps associated with the dislocation shear can be computed using Barnett formula optimized for DDD codes [52. The procedure allows to compute the actual shape of the free surface during the cycling.

The whole configuration of the free surface at the $26^{\text {th }}$ cycle, when the total plastic deformation is zero, is presented in Fig 4(a) and the colors indicate the value of displacement projected along the normal to the surface. From the profile line in Fig 4(c), extrusions and intrusions are clearly illustrated, which are the typical surface morphologies observed experimentally [15, 14, 53]. The evolution of maximal extrusion and intrusion heights as a function of the loading cycles are plotted in Fig 4 (d)). The two values increase during the formation process of the PSBs but after the $26^{\text {th }}$ cycle, the height values simply fluctuate around a constant. Fig 4(b) presents the local evolution of extrusions from cycle 22 to cycle 26 . At the $22^{\text {nd }}$ and $24^{\text {th }}$ cycle, there are only two layers of extrusions. Then, one layer is constructed again at the $26^{\text {th }}$ cycle. The growth of extrusions is induced by the passage of prismatic loops across the free surface [32] vacancy prismatic loops lead to intrusions and interstitial prismatic loops induce extrusions. Each prismatic loop contributes to one layer of extrusion or intrusion with a magnitude of one Burgers vector. The extrusions and intrusions growth is realized by the accumulation of these layers. It should be noted that the dimension of the layer depends also on the size of the prismatic loops. In other words, the height and morphology 
of the extrusion and intrusion are influenced by the character and shape of the prismatic dislocation loops crossing the free surface.
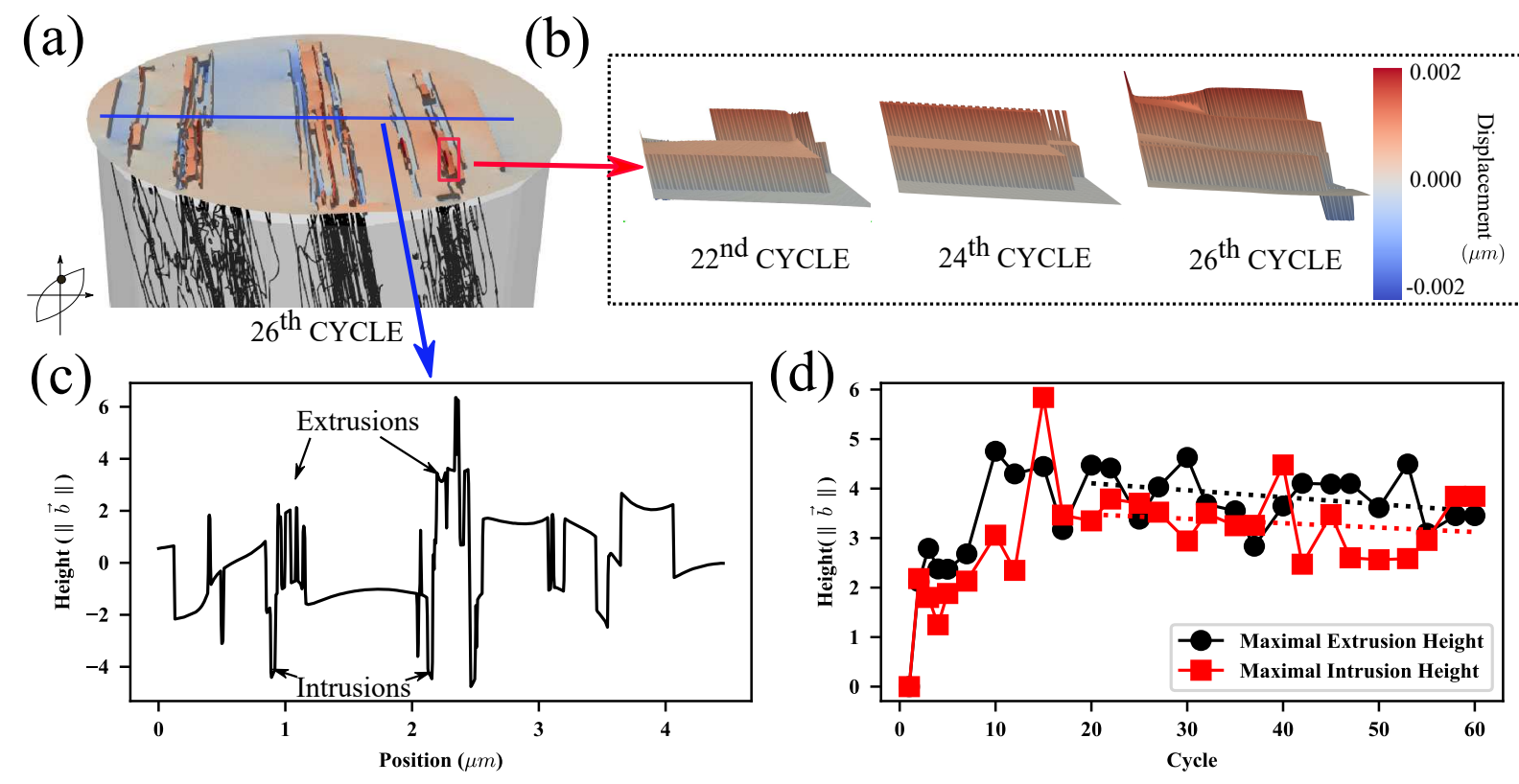

Figure 4: Roughness on the free surface of the grain when the global deformation is zero for simulation of a Cu cylindrical grain $(D=H=5 \mu m)$ under $\left(\varepsilon_{p, a p p l i e d}^{V M}=10^{-3}\right)$ : (a) global configuration of the surface with the corresponding dislocation microstructure at cycle 26; (b) zoom on the local growth of one extrusion during 4 cycles; (c) line profile at cycle 26 plotted along the diameter (blue line); (d) evolution of the maximal extrusions/intrusions heights with loading cycles.

\subsection{Analysis on the saturation regime}

As said before, a saturation state is reached after about 26 cycles as evidenced in Fig 3 . In this so-called saturation regime, even if the plastic deformation loading continues, many characteristics remain stable including the PSB number, PSB locations, PSB thicknesses, dislocation densities, and maximal height of surface extrusions and intrusions. It should also be pointed out that the saturation regime has not been reported by former DDD simulations of fatigue.

The stability of the dislocation density suggests that a dynamic equilibrium in the dislocation movements is reached, which implies that the number of dislocations created within a cycle is equal to the ones annihilated or escaping at the free surface. Moreover, the consistency of PSBs number and thickness imply that the dislocation-related microstructures do not change significantly in the saturation regime, that is to say the microstructure is stable. As the maximal height of extrusion and intrusion is correlated to the crossing of prismatic loops, the slight fluctuation of these two values demonstrates that the flux of prismatic loops nucleation inside the PSB is almost constant during the saturation regime. In other words, the dislocation nucleation kinetics has reached a permanent regime.

It should be emphasized that saturation phenomenon has been widely observed in fatigue experiments conducted on FCC metals. The most famous experiments are the ones of Mughrabi on the single slip fatigued single crystal of $\mathrm{Cu}[4,8$ : under the cyclic loading, PSBs are formed and result in a rapid hardening. The observation of specimen surfaces has demonstrated that the PSBs are stable once they are well constructed: the position, the thickness and the volume fraction remain almost unchanged, which corresponds to the simulation outcome (See Fig 33). This characteristic seems to be a constant among FCC metals. Pure Ni single crystal has shown the same behavior as $\mathrm{Cu}$ in the research work of Mecke [54]. Lavenstein et al. have also confirmed the saturation of the number of PSBs with the cycling [17. 
Huang et al. 55 has used in situ neutron diffraction to detect the dislocation density in a Ni-based super-alloy. The saturation of the dislocation density was observed: dislocation density increases with the cycles and saturates when the PSBs are formed. Essmann and Mughrabi [56] have used a simple model of dislocation accumulation as a function of the plastic strain to study the importance of dislocation annihilation and concluded that the annihilation of dislocations can lead to a natural limit of the dislocation density under cyclic loadings. The saturation of the dislocation density in our simulation suggests the same idea that an upper limit of stored dislocations exists for a given value of the imposed plastic strain amplitude.

Regarding the surface roughness, it is generally admitted that the height of extrusions or intrusions continuously increases and will ultimately lead to the initiation of cracks at room temperature. Compared with the model of Polák 15, 14 for the formation of surface extrusions, the growth of extrusions in our simulation can be considered as the so-called rapid growth of static extrusions because it is induced by the dislocation motion and the crossing of prismatic loops. In their model, the second stage of extrusion or intrusion growth is called stable growth [15, 14] and is associated to the diffusion of vacancies. Since the effect of vacancy diffusion is not included in our model, that may be the reason for the saturation of extrusion and intrusion heights in the DDD simulation. Moreover, the saturation in the PSB surface roughness has been reported by Mughrabi 57 especially at low temperature and with vacuum environment. The low temperature has prevented the diffusion of vacancies while the vacuum environment can reduce the irreversibility introduced by the oxidation at the surface. These experimental conditions match well the assumptions made in the DDD simulations which may be another explanation for the observed saturation phenomenon on the surface.
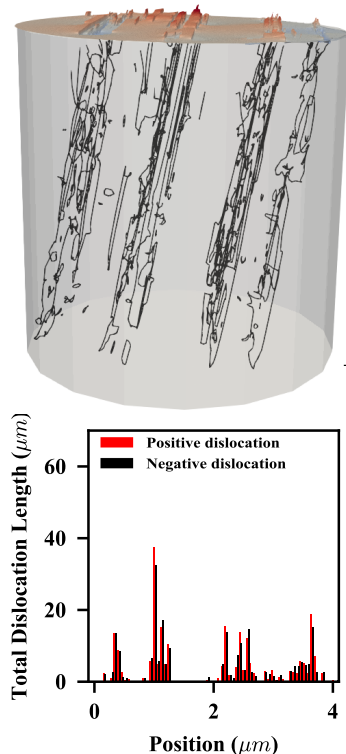
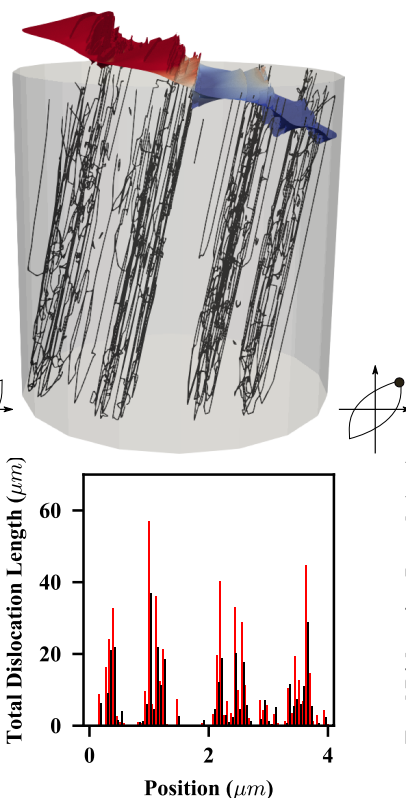
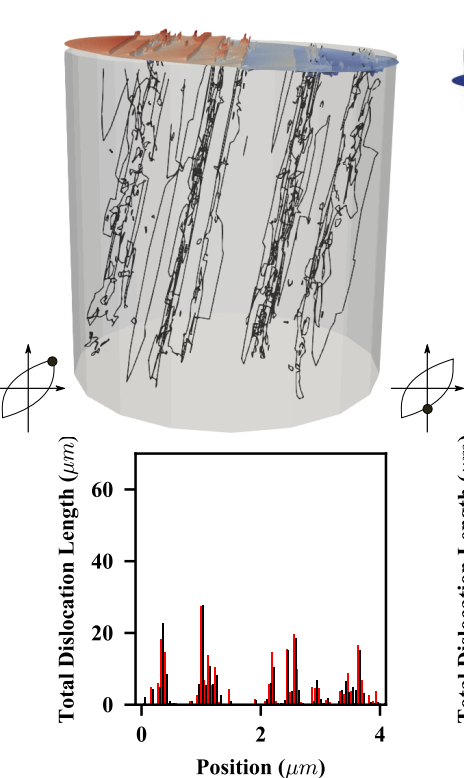
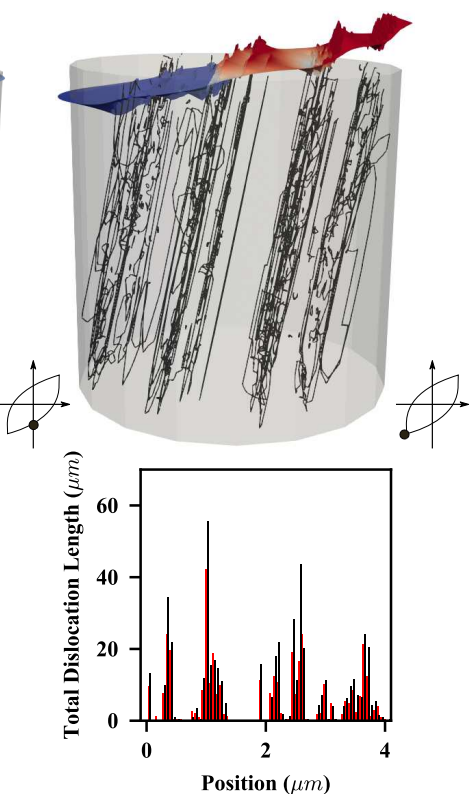

Figure 5: Surface deformation and evolution of dislocation distributions at the $50^{\text {th }}$ simulated cycle for different value of stress and strain in the loading cycle $(D=H=5 \mu \mathrm{m})$ under $\varepsilon_{p, \text { applied }}^{V M}=10^{-3}$ (the deformation has been amplified 300 times for clarity).

In experiments, the slip-path of dislocations within PSBs has attracted many research interests such as the study comparing the rhythmical and randomly oriented flip-flop in loop patches 58 . Generally, it is thought that dislocations move in the PSBs in an energy-favored way to accommodate plastic deformation [59, 60. One of the models is the gliding of screw dislocations in the channels and bowing out of edge dislocations from the walls [16. In order to understand the dislocation slip-path required to accommodate the plastic deformation during the saturation regime of the simulation, we have shown in Fig 5 the dislocation distribution evolution and the related surface deformation during the $40^{\text {th }}$ cycle. Snapshots and dislocation 
length analyses are performed at four instants of the cycle corresponding to the zero and maximal plastic deformations. First of all, during one cycle, the cyclic shear deformation is well simulated as evidenced by the changes in the surface shape during the cycle. The dislocation density also fluctuates with the variation of the applied deformation. It is mainly the dislocations in the two sides of the PSBs that multiply to accommodate the plastic deformation and the sign of increasing dislocations alternates with the direction of the applied load. Combined with the observation made in Fig 3(c), it can be inferred that in the simulation, the plastic deformation is mainly accommodated by the construction and destruction of pile-ups which are located at the interfaces between the PSBs and the matrix so that during the cyclic loading, the dislocation dipoles and prismatic loops located in the center of the PSBs are more stable and do not impede the pile-ups movements.

Dislocations move on slip planes (primary and cross-slip planes), driven by the corresponding shear stresses. According to the geometric relation between the simulated grain and the slip plane orientations (see Fig 1), dislocations which slip on the primary slip system mainly lead to the formation of pile-ups, dipoles in the PSB and dislocation crossing the free surface, while the movements in the transverse direction of the crystal is contributed by the dislocations slipping on the cross-slip plane. The localization of dislocations in the PSBs is the critical consequence of these transverse movements. To further explore the reasons for the dislocation localization, the distribution of the effective shear stress resolved on the cross-slip plane, including both the internal stress produced by dislocations and the applied stress, is presented in Fig 6 for different cycles. As indicated in the illustration, the plane used for the calculation is perpendicular to both the primary slip plane and the cross-slip plane, i.e. the plane normal is aligned with the Burgers vector shared by the two slip systems $\boldsymbol{n}=[110]$. At the second cycle, the dislocations are distributed homogeneously enough but as indicated by the red box, most of the dislocations are bearing a very strong cross-slip shear stress, which means they are not in a stable position. Under the effect of the shear stress, dislocations will move in the cross-slip plane (in the transverse direction) during loading and unloading. Let recall here that the positive or negative moving direction is determined by the sign of the shear stress and also the sign of the dislocations. The shear stress on the cross-slip plane provides indirect information on the dislocation ability to interact so that from the $2^{\text {nd }}$ cycle to the $10^{\text {th }}$ cycle, dislocations show a tendency to repeal each other. That is to say, dislocations move towards the two opposite sides of the grain respectively to reduce the stress on the cross-slip plane, which enables the formation of two PSBs. As the dislocations are more isolated, the shear stress and hence interactions between the dislocations in the two PSBs get weaker. The continuing cyclic loading from $10^{\text {th }}$ cycle to the $26^{\text {th }}$ cycle allows more dislocations to move away from the two PSBs and be pushed away towards the grain outer surfaces, which contributes to the formation of four PSBs. At the same time, it can be observed that some dislocations are stored in the center of the PSBs (dislocation dipoles and prismatic loops) and the shear stress resolved on the cross-slip system becomes smaller. Finally, in the saturation regime, the cross-slip shear stress in the matrix between two PSBs is much reduced, which explains why the dislocations cannot further move in the matrix to form another PSB.

350 This can be considered as a key mechanism to clear the dislocations in the matrix. As a result, from the $26^{\text {th }}$ to the $50^{\text {th }}$ cycle, the number of PSBs and their positions remain constant.

We can conclude that the stress state induces a self-organization of the dislocations that lead to the localization and PSB formations. And reciprocally, this localization largely reduces the dislocation interactions and hence the shear stress on the cross-slip planes. It stabilizes the dislocation microstructure so that the dislocations can move in a so-called low-energy mode with less stress interactions to accommodate the plastic deformation, which explains the saturation state from the $26^{\text {th }}$ cycle to the $50^{\text {th }}$ cycle.

\subsection{Influence of the imposed plastic strain amplitude}

The influence of the imposed plastic deformation on the fatigue behavior for FCC metals has been widely studied in experiments [19, 12, 20]. The plastic deformation amplitude is thought to have a significant effect on the fatigue behavior, especially on the microstructure, the macroscopic response and the hardening rate. For Copper single crystals, a larger deformation will eventually create more PSBs 12 and a very brutal raise of the applied plastic strain amplitude is able to activate more slip systems and will modify the PSB microstructure into cells [18. In order to depict the effect of the imposed plastic strain amplitude, a simulation campaign is conducted on the same geometry (cylindrical grain $D=H=5 \mu m$ ) under five 


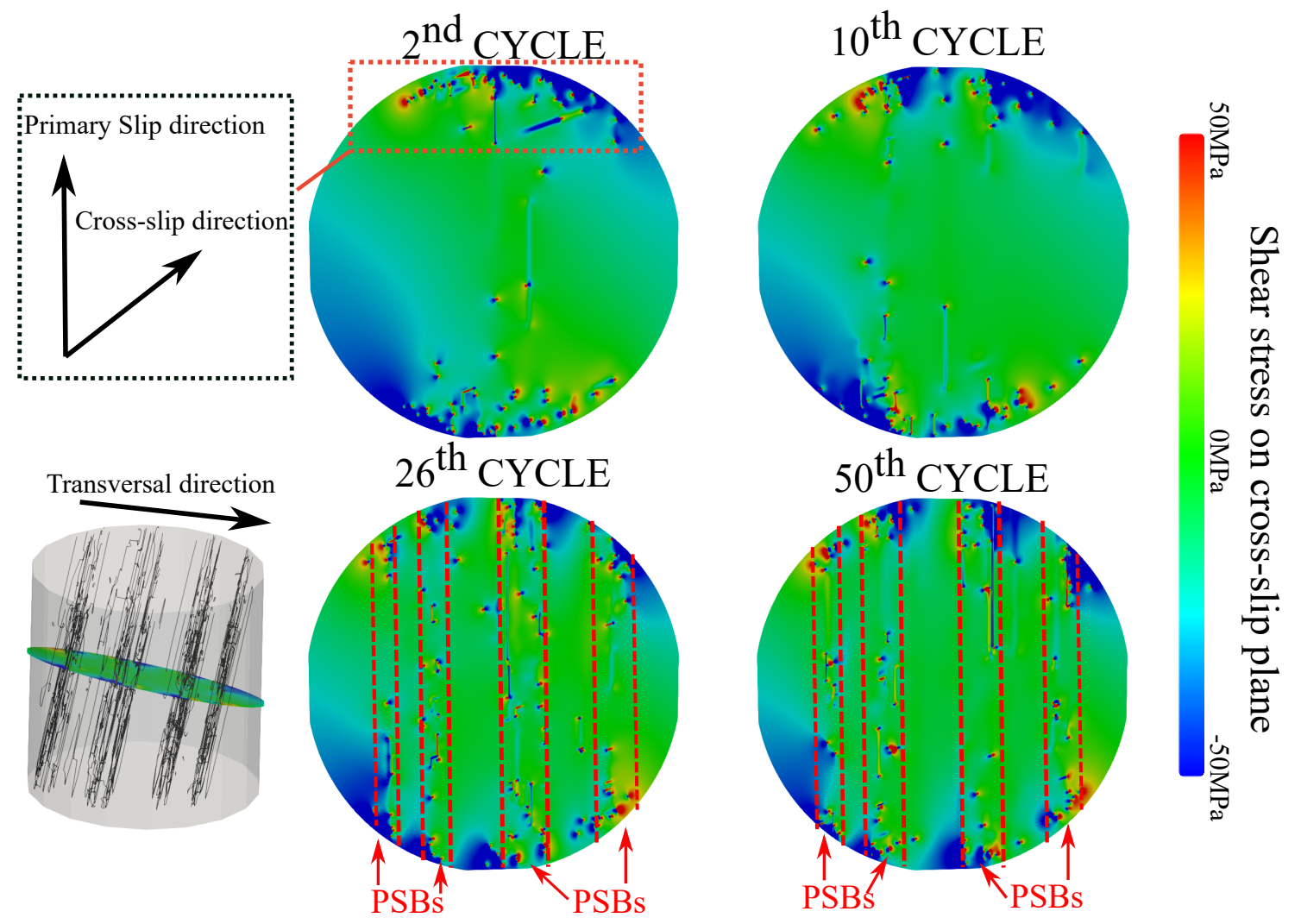

Figure 6: Evolution of the shear stress distribution on the cross-slip plane inside the grain with the formation of PSBs for simulation of $\mathrm{Cu}(D=5 \mu m$ and $H=5 \mu m)$ under $\varepsilon_{p, \text { applied }}^{V M}=10^{-3}$. 
different strain amplitudes $\left(\varepsilon_{p, a p p l i e d}^{V M}=0.6 \times 10^{-3}, 0.8 \times 10^{-3}, 1.0 \times 10^{-3}, 1.2 \times 10^{-3}\right.$ and $\left.1.5 \times 10^{-3}\right)$. Simulations are performed both for $\mathrm{Cu}$ and $316 \mathrm{~L}$. Fig 7 shows the evolution of the total dislocations densities for each of the five applied strain values for $\mathrm{Cu}$. Dislocation density increases along with $\varepsilon_{p, a p p l i e d}^{V M}$. Regardless of the value of the applied strain amplitude, and as seen in the previous simulation, after about 20 cycles of loading, the saturation regime is systematically reached. In fact, this stands not only for the dislocation densities, but also for the PSB height and the surface roughness.

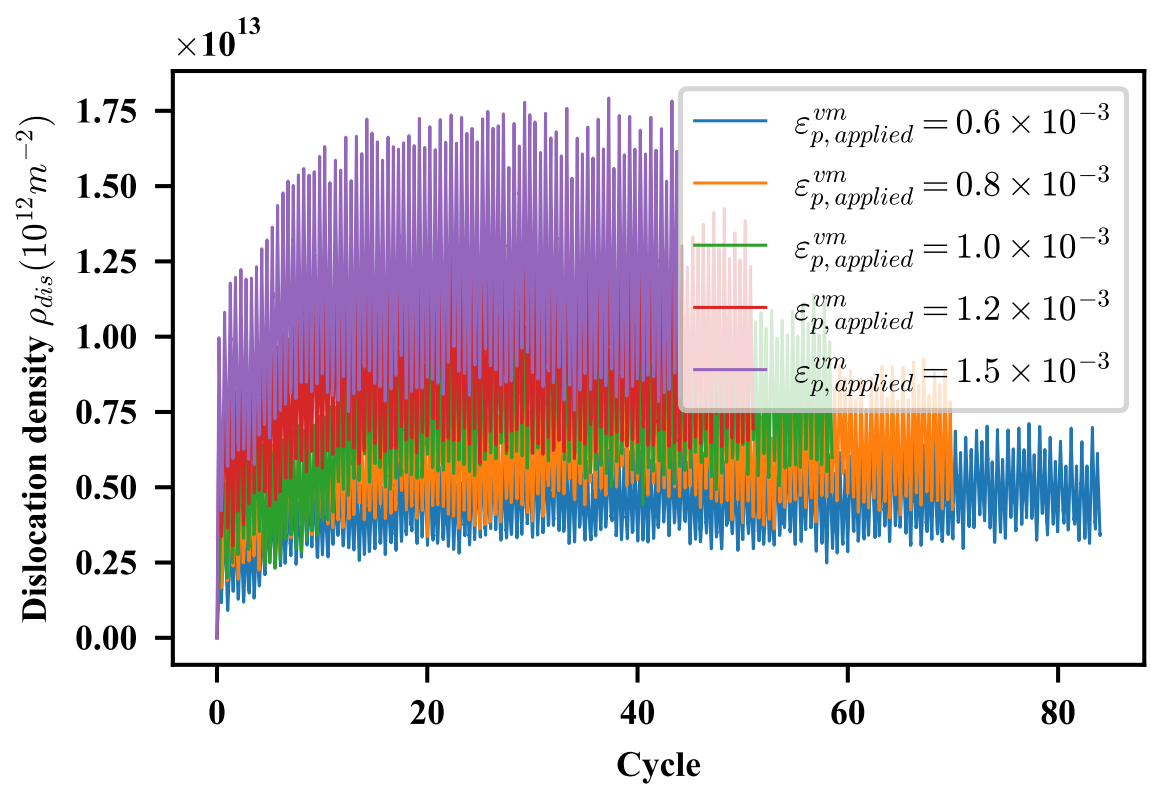

Figure 7: Evolution of the total dislocation density with the number of loading cycles for different applied strain amplitudes $(\mathrm{Cu}$, cylindrical grain $D=5 \mu m$ and $H=5 \mu m)$.

The dislocation microstructures obtained in the saturation regime (corresponding to the $40^{\text {th }}$ cycle) for $\mathrm{Cu}$ and $316 \mathrm{~L}$ are compared in Fig 8 for the different values of $\varepsilon_{p, \text { applied }}^{V M}$ and when $\varepsilon_{p}^{V M}=0$. From the 3D snapshots, it can be intuitively seen that a larger strain amplitude leads to more storage of dislocations as well as more construction of PSBs. It is worthwhile mentioning that for a given material, instead of increasing the individual PSB's thickness to accommodate the plastic deformation, more PSBs are constructed as if each PSB could only accommodate a finite amount of plastic strain. Comparing with $\mathrm{Cu}$, under the same deformation, 316L has constructed fewer PSBs.

Based on the linear relationship between the PSB volume fraction and the applied deformation for $\mathrm{Cu}$ in single slip mode, observed in experiments, Winter 12 has proposed a two-phases model to describe the relationship between the applied deformation and the formed PSBs. In his model, the plastic deformation is accommodated by the two phases separately: the first phase is the PSB, which is soft and active; the second one is the matrix, which is hard and almost inactive. The capacity of accommodation for the plastic deformation in these two phases are fixed. The relation is given as follows:

$$
\varepsilon_{p, \text { applied }}^{V M}=\varepsilon_{p, P S B} * f+\varepsilon_{p, \text { Matrix }} *(1-f)
$$

where $f$ is the volume fraction of PSBs in the grain and $\varepsilon_{p, P S B}, \varepsilon_{p, \text { Matrix }}$ are the plastic strain in the PSBs and the matrix respectively. This model has been applied successfully in many FCC metals such as $\mathrm{Cu}$ and $\mathrm{Ni}$ [12, 54 .

In order to verify if the evolution of the PSB volume fraction $(f)$ with the imposed plastic strain amplitude obtained in our simulations follows Winter's model, the PSB thicknesses are calculated using a method described in Fig 9. Firstly, the thickness is measured at maximal deformation, when many dislocation pile-ups are built-up so that the frontiers between PSBs are easier to identify. Fig 9(a) shows a typical 

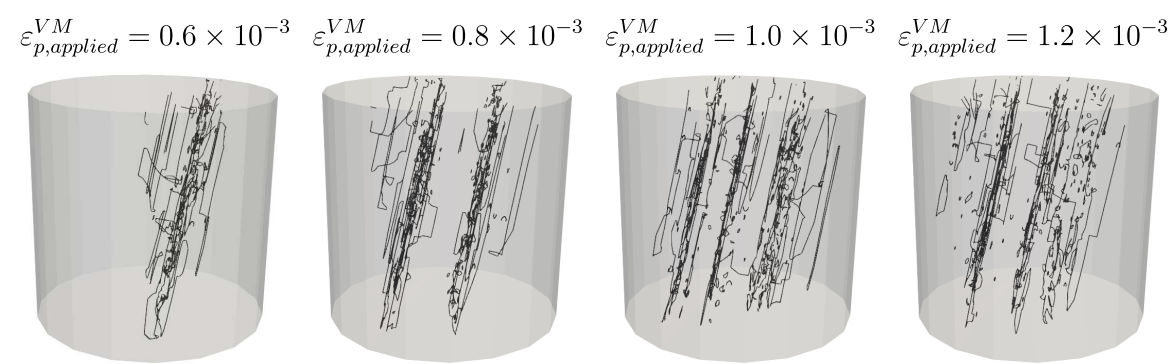

$\varepsilon_{p, \text { applied }}^{V M}=1.5 \times 10^{-3}$

316L
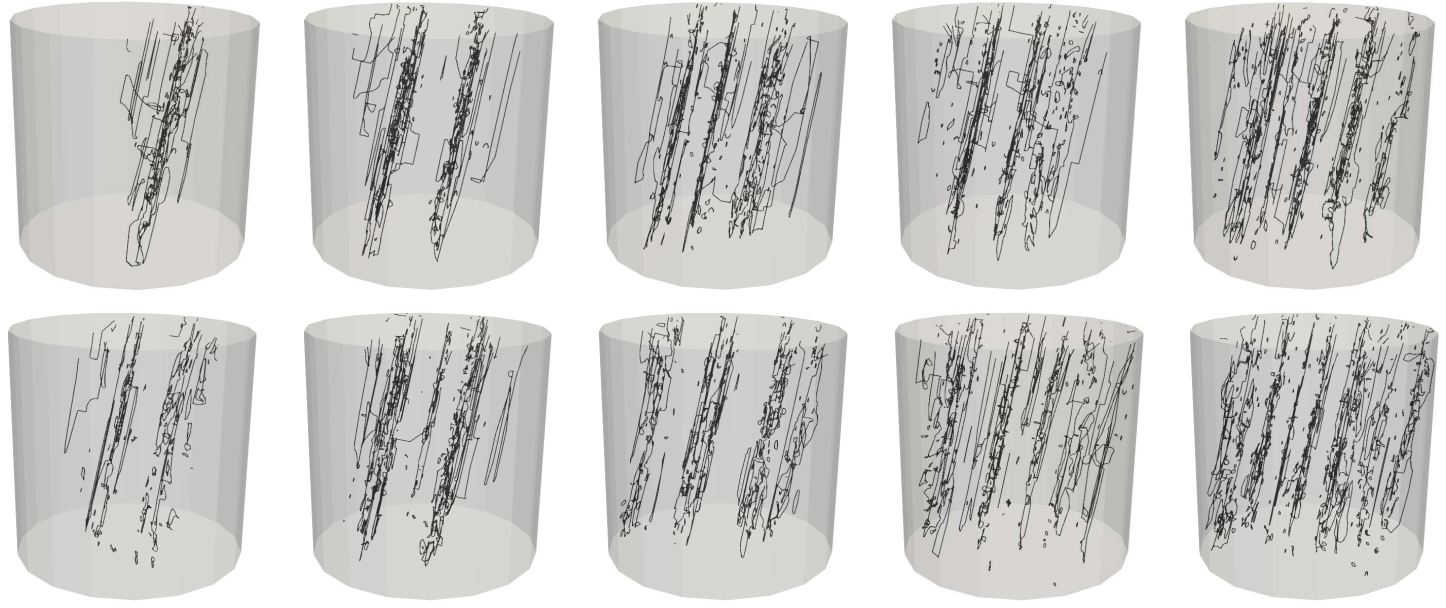

Figure 8: Comparison of the dislocation microstructures of $\mathrm{Cu}$ and $316 \mathrm{~L}$ at the cycle 40 for the different imposed plastic strain amplitudes (cylindrical grain, $D=H=5 \mu m$ ).

distribution of dislocations at maximal deformation for $316 \mathrm{~L}$ (cylinder grain with $D=5 \mu m$ and $H=5 \mu m$, under the applied deformation $\varepsilon_{p, \text { applied }}^{V M}=0.6 \times 10^{-3}$ ) taken at cycle 40 . For this situation, a single PSB is formed inside the grain. PSB line profiles, measured along five lines regularly spaced on the free surface and crossing the PSB, are plotted in Fig 9(b). Because of the non-zero deformation, the profiles are globally inclined. Note that the decrease of the profiles on the extremities comes from the numerical method used to calculate the surface displacement which is based on the calculations of the solid angle of the dislocation loops. The biggest steps visible on the profiles correspond to the local shear strain induced by dislocation pile-ups in the PSBs. By means of this property, as shown in Fig 9(b), the thickness of PSB is estimated from the length in the profile where the slope is maximum. The distribution of the total dislocation length along the position of the slip plane (Fig 9(c)) at maximal deformation and zero deformation is considered as a validation of thickness measurement and helps to get rid of the influence of isolated reversible dislocations.

The volume fraction of PSBs is then calculated as the ratio between the total PSB thicknesses and the grain diameter $D$. The relation found between the PSB volume fraction and the imposed plastic strain amplitude for $\mathrm{Cu}$ and $316 \mathrm{~L}$ is illustrated in Fig 10 . Since the matrix in our simulation contains almost no dislocations, $\varepsilon_{p, \text { Matrix }}$ is supposed to be zero so that the fitting function should write $\varepsilon_{p, a p p l i e d}^{V M}=\varepsilon_{p, P S B} * f$. As it can be seen Fig 10, such a linear relationship matches well the evolution of the PSB thickness with strain measured from our DDD simulations. The slope of the fitting linear equation gives the value of $\varepsilon_{p, P S B}$ for $\mathrm{Cu}$ and $316 \mathrm{~L}$. It is concluded that the capacity of a PSB to accommodate plastic strain for 316 $\left(\varepsilon_{p, P S B}=5.055 \times 10^{-3}\right)$ is bigger than that of $\mathrm{Cu}\left(\varepsilon_{p, P S B}=2.945 \times 10^{-3}\right)$. The comparison between the 410 behaviors of $\mathrm{Cu}$ and $316 \mathrm{~L}$ is discussed in the last section.

In brief, for the first time, the linear relationship between the volume fraction of PSBs $(f)$ and the applied plastic strain is confirmed by DDD simulations. Such a relationship means that the formed microstructure in the PSBs has a fixed capacity to accommodate plastic deformation. Consequently, the PSBs adjust their volume fractions according to the imposed plastic strain amplitude.

\subsection{Stability of the PSBs}

Winter has constructed its model by analogy with the liquid-vapor system but there is one limitation: the transformation from vapor to liquid or from liquid to vapor is perfectly reversible while the constructed PSB cannot have such a high level of reversibility. Winter has confirmed this limitation by realizing experiments with a decreasing loading amplitude and observed surface slip lines of PSB. He concluded that few PSBs 


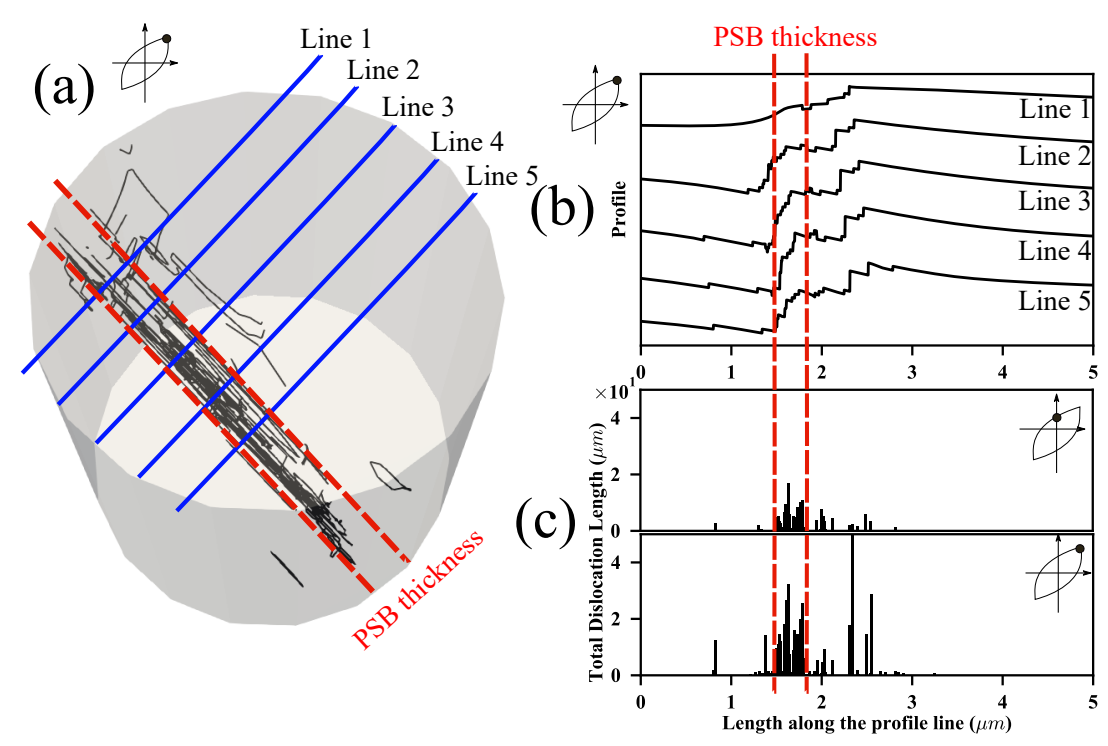

Figure 9: Illustration of PSB thickness measurement: (a) Dislocation microstructure (PSB) inside the crystal with five profile lines at the free surface; (b) PSB line profiles at maximal strain; (c) dislocation distribution histograms at zero strain and maximum strain $\left(316 \mathrm{~L}\right.$, cylindrical grain, $\left.\varepsilon_{p, \text { applied }}=0.6 \times 10^{-3}, D=5 \mu \mathrm{m}, \mathrm{H}=5 \mu \mathrm{m}\right)$.

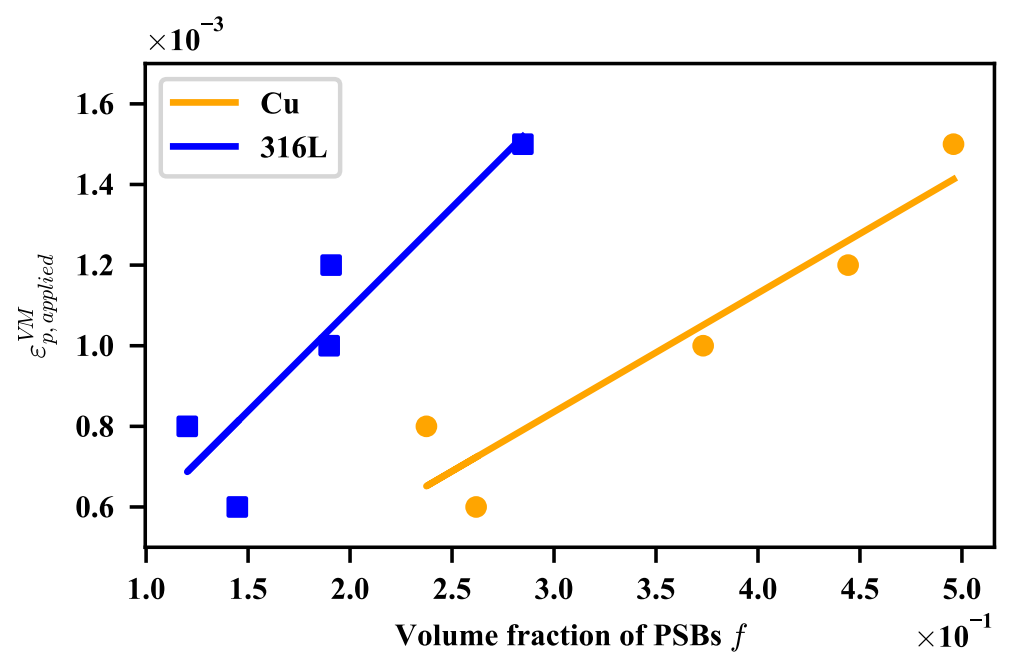

$$
\begin{aligned}
\mathrm{Cu}: \varepsilon_{p, \text { applied }}^{V M} & =2.945 \times 10^{-3} * f-4.769 \times 10^{-5} \\
316 \mathrm{~L}: \varepsilon_{p, \text { applied }}^{V M} & =5.055 \times 10^{-3} * f+7.957 \times 10^{-5}
\end{aligned}
$$

Figure 10: Linear relationship between the volume fraction of PSB, $f$, and the applied strain amplitude $\varepsilon_{p, a p p l i e d}^{V M}$ for both Cu and 316L (cylindrical grain $D=H=5 \mu m$ ). 
constructed at a high deformation amplitude survive when the amplitude is decreased but becomes inactive, which proves the irreversibility of the PSB microstructure. In order to investigate the irreversible character of PSBs formed in our DDD simulations, a two steps fatigue loading is applied on the Cu single crystal $(D=H=5 \mu m)$. During the first 25 cycles, a plastic strain amplitude of $\varepsilon_{p, \text { applied }}^{V M}=1.2 \times 10^{-3}$ is applied and then reduced to $\varepsilon_{p, \text { applied }}^{V M}=0.6 \times 10^{-3}$. The obtained stress-strain curve is plotted in Fig 11(a). During the second part of the loading, when the applied strain is reduced by a factor 2 , the dislocation organization mechanism is affected by the prior construction of the PSBs during the first 25 cycles and as the applied deformation decreases, the initial microstructure contains more PSBs than necessary to accommodate the plastic deformation. As shown in Fig 11(b), the dislocation density drops down shapely at the $26^{\text {th }}$ cycle and decreases gradually in the subsequent 15 cycles to attain a new saturation regime. It is found that the decrease of a factor 2 in the applied strain amplitude leads to a decrease of a factor 2 in the dislocation density measured in the respective saturation regimes.
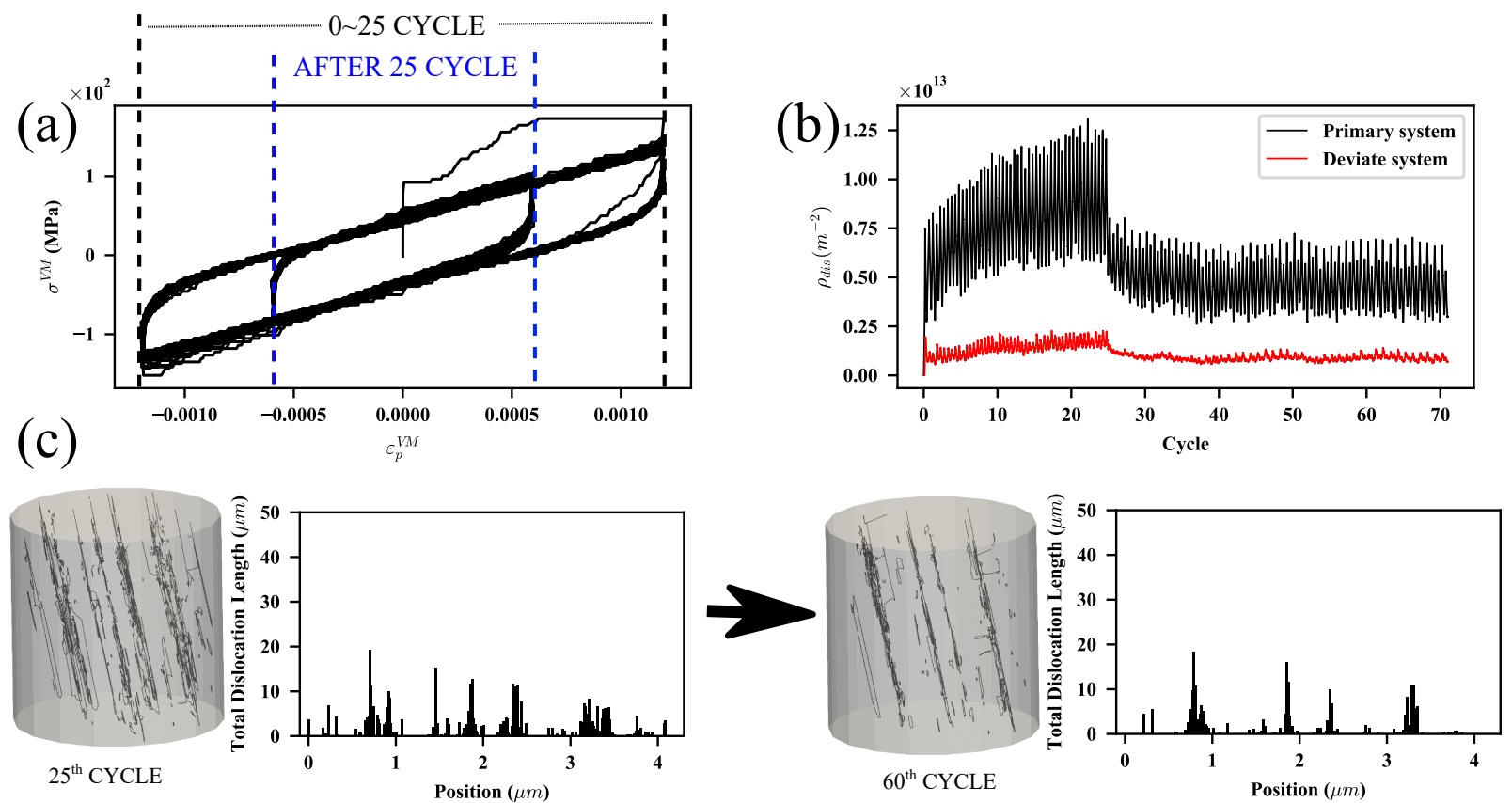

Figure 11: (a) Stress-strain curve for the simulation with decreasing applied deformation, the first 25 cycles are applied with $\varepsilon_{p, \text { applied }}^{V M}=1.2 \times 10^{-3}$ then the loading amplitude is reduced to $\varepsilon_{p, \text { applied }}^{V M}=0.6 \times 10^{-3}$; (b) dislocation density evolution for primary and deviate slip systems with the loading cycles; (c) the dislocation distribution evolution when the applied deformation is reduced from $\varepsilon_{p, \text { applied }}^{V M}=1.2 \times 10^{-3}$ ) at the $25^{\text {th }}$ cycle to $\varepsilon_{p, \text { applied }}^{V M}=0.6 \times 10^{-3}$ at the $60^{\text {th }}$ cycle and the corresponding distribution histogram ( $\mathrm{Cu}$, cylindrical grain, $D=5 \mu m, H=5 \mu m)$.

An interesting feature concerns the evolution of the PSB microstructure distribution. In Fig 11 (c), the number of PSBs in the last cycle before the strain drop is about 6 (two smaller PSBs and four thicker PSBs). The thicknesses of the PSBs are clearly presented in the dislocation distribution diagrams. After 35 cycles continuing the lower deformation loading: the dislocation density decreases and the thickness of each PSB also becomes thinner. On one hand, the four thicker PSBs stay at the same position after the reduction of loading, on the other hand, the two thinner PSBs have almost disappeared and only a few debris remains.

Winters model has been cited in many publications but the fatigue test with a decreasing loading is rarely tried in practice. Blochwitz [10] has tested the evolution of PSBs with a decreasing loading for Ni and he has observed the changes induced on the PSBs. Fig 12 shows the main experimental results of this publication. The position of macro-bands developed at the plastic amplitude $\varepsilon_{p}=3.0 \times 10^{-3}$ in the saturation regime can be observed in Fig 12 (a) while Fig 12 (b) displays the macro-bands developed at the same sites after a decrease of the strain amplitude to $\varepsilon_{p}=1.0 \times 10^{-3}$ until another saturation regime. By 
comparing the old and the new bands, the authors concluded that the positions of macro-bands at the higher and lower amplitude correspond well to each other, however, at the lower amplitude, the intensity of the bands is smaller than at higher amplitude.

(a)

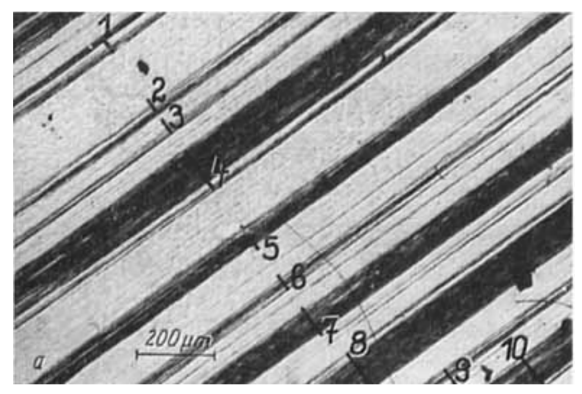

(b)

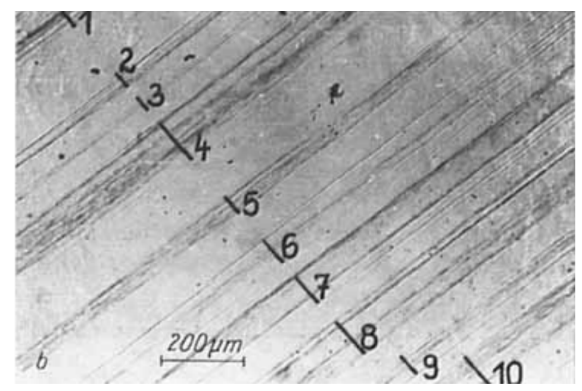

Figure 12: Comparison of formed macro-band position: (a) the positions at high amplitude $\varepsilon_{p}=3.0 \times 10^{-2}$; (b) the positions at lower amplitude $\varepsilon_{p}=1.0 \times 10^{-2}$ from [10] with permissions.

Our DDD simulations could reproduce these experimental results, especially, for the four thicker PSBs which stay at the same positions, but reduces in thickness after reduction of the plastic strain amplitude. Similarly, the debris observed at the lower applied strain remain at the same location as the PSBs formed during the high plastic strain loading. Even though the thickness scale of the experimental PSBs $(\sim 10 \mu m$ from Fig 12$)$ and the numerical PSBs $(\sim 1 \mu \mathrm{m}$ from Fig 3$)$ in DDD is different, the interpreted similarity can be considered to be qualitative.

\subsection{Comparisons between $C u$ and $316 L$}

We have already mentioned in the discussions above that the fatigue behaviors of $\mathrm{Cu}$ and AISI 316L austenitic stainless steel are different. In Fig 8, under the same deformation amplitude, evidently, the distribution of dislocations for $\mathrm{Cu}$ and $316 \mathrm{~L}$ is different. As an example, for $\varepsilon_{p}^{V M}=0.6 \times 10^{-3}, \mathrm{Cu}$ has formed two PSBs while 316L has only constructed one PSB occupying less volume than $\mathrm{Cu}$. The simulations have shown that this is a general trend: for a given plastic strain amplitude, $\mathrm{Cu}$ builds up more PSBs which occupy a larger volume fraction of the grain than 316L. From the fitting equation of Fig 10. we have concluded that 316L's PSBs have a higher capacity to accommodate the plastic deformation. A more quantitative comparison is now plotted in Fig 13 in terms of the thicknesses of the PSBs for the two materials. It can be seen that under the same strain amplitude, $\mathrm{Cu}$ is more likely to construct a thicker PSB. The distribution of individual PSB thickness in $\mathrm{Cu}$ is more dispersed than that in 316L. In fact, the dislocation density in the two materials is almost identical in the saturation regime. However, the dislocation 5 arrangement in $\mathrm{Cu}$ is less compact than in 316L.

To understand the observed results, the PSB formation mechanism should be further investigated. As observed in the simulation and mentioned in [45, dislocation glide on the primary slip plane is dominant and dislocation glide on the deviate plane is minor but crucial for the deformation spreading in the transverse direction and for the formation of PSBs. Finally dislocation multiplication occurs in the crystal through a double cross-slip mechanism which consists of two consecutive cross-slip occurrences: from primary plane to deviate plane, then from deviate plane to primary plane. It can be inferred that the character of cross-slip plays a critical role in the dislocation distributions.

The probabilities for cross-slip in our model for $\mathrm{Cu}$ and 316L are plotted in Fig 14(a). Evidently, under the same shear stress $\tau_{d e v}, \mathrm{Cu}$ has a higher probability of cross-slip. As indicated by the value given to $\tau_{I I I}$ in Tab 2, cross-slip is triggered for a lower stress value for $\mathrm{Cu}(32 \mathrm{MPa})$ than for $316 \mathrm{~L}(56 \mathrm{MPa})$ but this should be scaled by the elastic constant of the investigated materials. The simple ratio $R_{c s}=\frac{\tau_{I I I}}{\mu}$ can be defined to compare the cross-slip activities in the two materials. It is remarkable that this ratio is comparable for both materials: $R_{c s}^{C u} / R_{c s}^{316 L}=1.03$ while the fatigue behavior is different.

All simulations for $\mathrm{Cu}$ and $316 \mathrm{~L}$ starts with a single initial dislocation source. The primary microstructure to be constructed in the first cycle is one individual dislocation pile-up. The first cross-slip occurs in the 


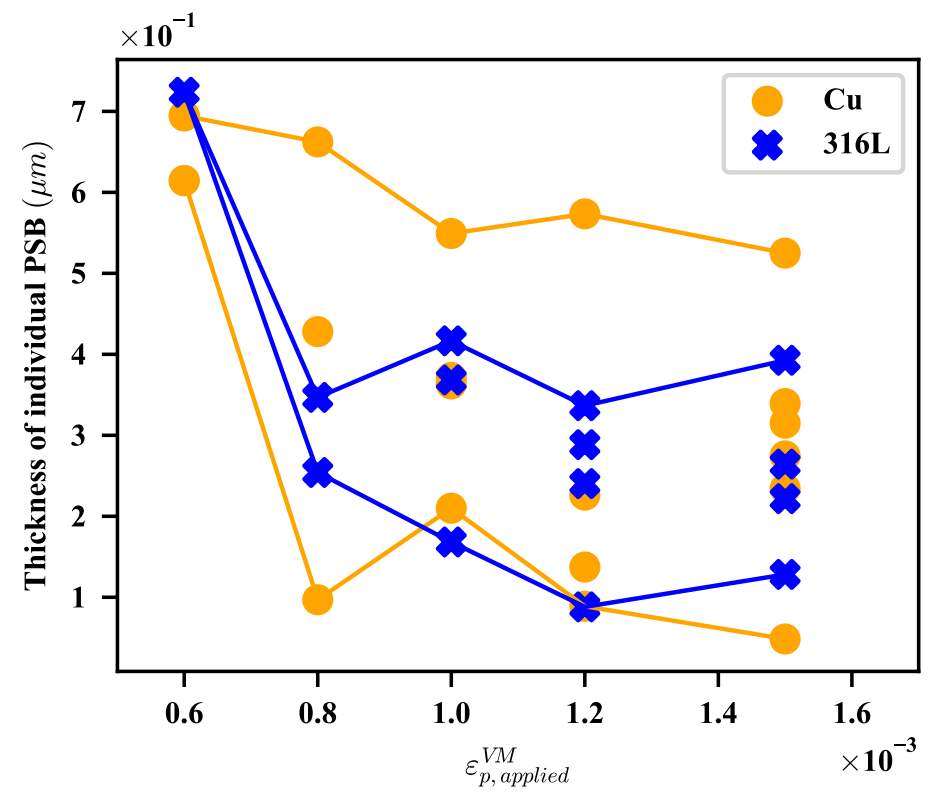

Figure 13: Thicknesses of each PSB under different imposed strain amplitude for $\mathrm{Cu}$ and $316 \mathrm{~L}$ (cylindrical grain, $D=H=$ $5 \mu m)$.

pile-up for $\mathrm{Cu}$ when the applied plastic deformation is $\varepsilon_{p}^{V M}=0.25 \times 10^{-3}$ while in $316 \mathrm{~L}$, the first cross-slip happens at $\varepsilon_{p}^{V M}=0.65 \times 10^{-3}$ : it reflects also a higher possibility of cross-slip in $\mathrm{Cu}$.

During the whole simulation, a good indicator of the cross-slip activity is the number of slip plane changes in the microstructure. The evolution of this parameter with the number of cycles is plotted in Fig $14(\mathrm{~b})$. It is shown that the number of slip plane changes is increasing with the number of loading cycles. And for larger deformation, slip plane changes are more frequent. As expected, we observed that generally, for a given deformation, the number of slip plane changes in $\mathrm{Cu}$ is more important than in 316L. Even though this parameter doesn't distinguish what is cross-slip events and what is line recombinations, it proves fairly well that $\mathrm{Cu}$ presents the characteristic to trigger more cross-slip events than $316 \mathrm{~L}$.

The relatively higher cross-slip frequency of $\mathrm{Cu}$ has several consequences. Primarily, the mobility of dislocations is significantly enhanced. Because of this, dislocations in $\mathrm{Cu}$ can explore a larger volume which explains a higher possibility for $\mathrm{Cu}$ to form wide PSBs. Secondly, the easiness of cross-slip promotes the creation of more dislocation sources via the Koehler mechanism. More initial dislocation sources lead to more pile-ups as well as more PSBs to share the accommodation of the plastic deformation. Since the total plastic strain is fixed, the average plastic deformation accommodated by every pile-ups is smaller, which means that in $\mathrm{Cu}$, fewer dislocation loops are contained in the piled-up zones. The number of pile-ups in one PSB is limited to a maximum of 2 per PSB (on the 2 sides). Thus, for $\mathrm{Cu}$, the reduced number of dislocation loops in pile-ups limits the capacity of PSBs to accommodate the imposed strain so that more PSBs are required. It can be concluded that cross-slip easiness can be considered as the origin of the differences in the fatigue behaviors of $\mathrm{Cu}$ and $316 \mathrm{~L}$.

The cross-slip ability of 316L and $\mathrm{Cu}$ can also be deduced from the analysis of the shear stress resolved on the cross-slip system during the saturation regime (see Fig 15). As previously discussed in Fig 6, after the formation of four PSBs in $\mathrm{Cu}$, the dislocations are well stabilized in the grain and the shear stress in the cross-slip system is very small. However, from the distribution of shear stresses on $316 \mathrm{~L}$, it can be seen that the shear stress on the cross-slip plane is still really high, at least bigger than the one of $\mathrm{Cu}$, which means the dislocations in PSBs of 316L are likely to cross slip. Furthermore, dislocations in 316L have not moved to another place for self-stabilization. It is because in 316L, cross-slip requires much larger shear stress, even though the shear stress near the PSBs is so strong, dislocations in the PSB can not slip on the 
(a)

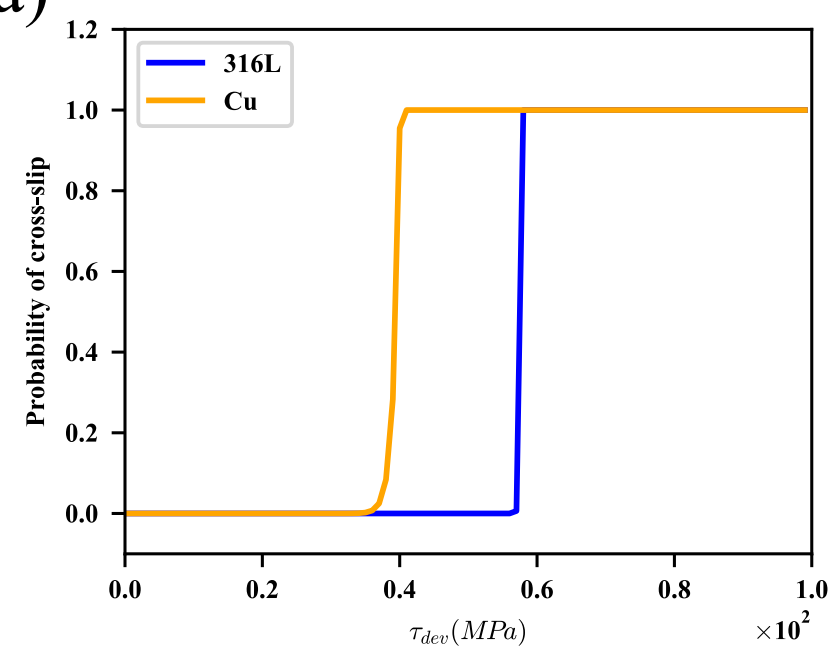

(b)

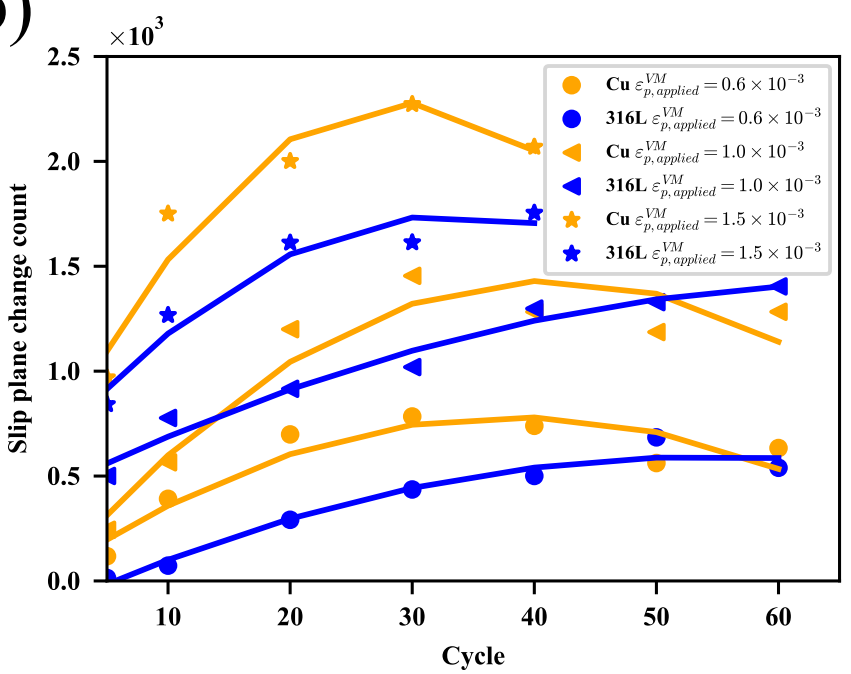

Figure 14: (a) Probability of cross-slip in the DDD model for $\mathrm{Cu}$ and 316L; (b) evolution of the count of slip plane changes for $\mathrm{Cu}$ and $316 \mathrm{~L}$ under different imposed plastic strain amplitude with the loading cycles. 
cross-slip plane to seek for a more stable position.

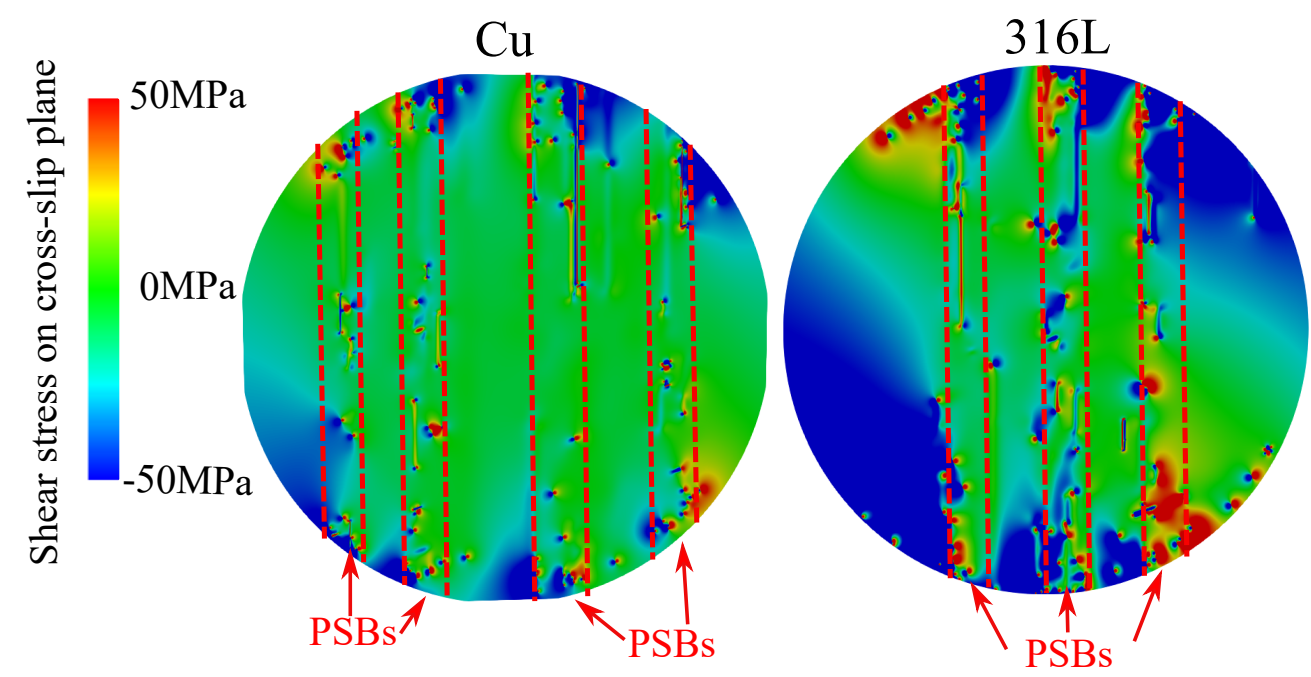

Figure 15: Comparison of the shear stress resolved on the cross-slip plane in $\mathrm{Cu}$ and $316 \mathrm{~L}$ at the $50^{\text {th }}$ cycle (Cylindrical grain, $\left.D=5 \mu m, H=5 \mu m, \varepsilon_{p}^{V M}=0.6 \times 10^{-3}\right)$.

Since the cross-slip probability is correlated to the stacking fault energy (SFE), the slip mode in fatigue is strongly determined by the SFE as reported in literatures [48, 50, 61, 62. For high SFE materials, like $\mathrm{Cu}$, the slip mode is called wavy slip, while for low SFE, like $\mathrm{Cu}-16$ at\% $\mathrm{Al}$ alloy, the slip mode is called planar slip. The dislocation microstructure of planar slip shows dislocations confined in planes marked by dipole arrays whereas wavy slip materials prefer to construct a more three-dimensional microstructure where dislocations aggregate to form veins or walls. Even though veins or wall have not been simulated in this work, our simulation results for $\mathrm{Cu}$ and 316L correspond well to these experimental observations: since $\mathrm{Cu}$ has a higher SFE $\left(80 \mathrm{MJ} / \mathrm{m}^{2}\right)$ than $316 \mathrm{~L}\left(30 \mathrm{MJ} / \mathrm{m}^{2}\right)$, Cu should construct a more 3D structure, which is what we have obtained in our simulations: $\mathrm{Cu}$ constructs PSBs occupying more volume fraction than 316L. The 3D character is not evidenced by our simulations which were performed in single slip loading conditions, but surely a different loading state such as a double glide configuration, would lead to a more three-dimensional geometry for $\mathrm{Cu}$ than for $316 \mathrm{~L}$.

\section{Concluding remarks}

Three dimensional Discrete Dislocation Dynamic simulations have been conducted on a cylindrical surface grain of $\mathrm{Cu}$ to investigate the formation of PSBs and the involved mechanisms. A detailed analysis of the PSB formation process, the influence of the imposed plastic strain amplitude and the comparison between $\mathrm{Cu}$ and $316 \mathrm{~L}$ behaviors, have led to the following conclusions :

- Localization of dislocations is observed in the simulations and results in the formation of slip bands, which are constituted mainly by dislocation dipoles and prismatic loops. These slip bands correspond to embryo of PSBs. Dislocation pile-ups are evidenced at the interface between the PSBs and the matrix. These pile-up dislocations accommodate most of the imposed plastic deformation. The mechanism of formation of the PSBs is highly related to the shear stress distributions in the cross-slip plane.

- After a few cycles, a saturation regime is reached: the average dislocation densities, the PSB volume fraction, PSB location as well as extrusions and intrusions heights are stable. This saturation phenomenon is also observed experimentally in Copper surface grains. 
- The effect of the imposed plastic strain amplitude on the PSB volume fractions verifies the Winter two-phases model. To the best of the author's knowledge, this is the first time that this model is validated by DDD simulations.

- By reducing the imposed strain amplitude, the degree of reversibility of formed numerical PSBs is verified. It is stated that the position of formed PSBs remains unchanged, which is also predicted by Winter's model.

- When comparing similar simulations realized on two materials chosen for their difference in their stacking fault energy $(\mathrm{Cu}$ and $316 \mathrm{~L})$, it is found that $\mathrm{Cu}$ constructs more PSBs and has a higher tendency to build up thicker PSBs. Also, the capacity of the PSBs in $\mathrm{Cu}$ to accommodate plastic deformation is found to be weaker than in AISI 316L. The differences of cyclic behaviors are thought to be induced by a different cross slip ability. The easiness of cross-slip in Cu leads to a higher mobility of the dislocations which, on one hand, facilitates the PSBs construction and augmentation of PSB's thickness, and on the other hand, reduces the dislocations loops localized in the pile-ups, which reduces the plastic accommodation capacity.

Although most of the main characteristics of fatigue at the early stages are well reproduced by the simulations presented here, DDD application to fatigue still has some weakness. In experiments, the observation is realized at the surface of samples where the oxidation may contribute to the slip irreversibility of the bands, which can deepen the slip lines at the surface and eventually modify the extrusion growth rate. In our DDD simulations, the oxidation process at the free surface has not been considered such as many other factors like precipitation and forest hardening from the other slip systems, which can all contribute to the irreversibility in dislocation motion.

For forthcoming simulations of fatigue, the irreversibility in DDD simulations should be reconsidered. Because in a monotonic loading process, the irreversibility is not as important as in cyclic loading situation, it has often been neglected in DDD modeling of monotonic simulations. In fatigue simulations, the irreversibility is introduced mainly by cross-slip, dipole formations and line recombinations but if the deformation is too small, the dislocations cannot be stored due to the lack of irreversibility. This result is different from the experimental observations, in which the irreversibility can always be brought even in the case of high-cycle fatigue. The augmentation of the friction stress is a possible way to augment the irreversibility based on arguments from solid solution contents. The add of precipitations or secondary slip systems can also be a solution. To conclude, the current DDD model can still be optimized to better simulate dislocation interactions, typically for high cycle fatigue simulations.

\section{Acknowledgments}

The authors gratefully acknowledge the 4-year PhD scholarship support from China Scholarship Council (CSC).

\section{References}

[1] J. Schijve, Fatigue damage in aircraft structures, not wanted, but tolerated?, International Journal of Fatigue 31 (6) (2009) 998-1011. doi:10.1016/j.ijfatigue.2008.05.016

[2] S. Courtin, High cycle thermal fatigue damage prediction in mixing zones of nuclear power plants: engineering issues illustrated on the father case, Procedia Engineering 66 (2013) 240-249. doi:10.1016/j.proeng.2013.12.079

[3] C. L. Atwood, V. N. Shah, W. J. Galyean, Analysis of pressurized water reactor primary coolant leak events caused by thermal fatigue, Tech. rep., Idaho National Engineering and Environmental Lab., Idaho Falls, ID (US) (1999).

[4] S. Suresh, Fatigue of materials, Cambridge university press, 1998.

[5] A. Wöhler, Über die festigkeitsversuche mit eisen und stahl, Ernst \& Korn, 1870.

[6] P. Forsyth, Fatigue damage and crack growth in aluminium alloys, Acta Metallurgica 11 (7) (1963) 703-715. doi: 580 /10.1016/0001-6160(63) 90008-7

[7] J. A. Ewing, J. Humfrey, Vi. the fracture of metals under repeated alternations of stress, Philosophical Transactions of the Royal Society of London. Series A, Containing Papers of a Mathematical or Physical Character 200 (321-330) (1903) 241-250. doi:10.1098/rsta.1903.0006 
[8] H. Mughrabi, The cyclic hardening and saturation behaviour of copper single crystals, Materials Science and Engineering 33 (2) (1978) 207-223. doi:10.1016/0025-5416(78)90174-X

[9] Y. Li, C. Laird, Cyclic response and dislocation structures of aisi 3161 stainless steel. part 2 polycrystals fatigued at intermediate strain amplitude, Materials Science and Engineering A 186 (1-2) (1994) 87-103. doi:10.1016/0921-5093(94) 90307-7

[10] C. Blochwitz, E. Kahle, A method for the determination of the cyclic stress-strain curve of presistent slip bands in fatigued single crystals, Kristall und Technik 15 (8) (1980) 977-986. doi:10.1002/crat.19800150818

[11] N. Thompson, N. Wadsworth, N. Louat, Xi. the origin of fatigue fracture in copper, Philosophical Magazine 1 (2) (1956) 113-126. doi:10.1080/14786435608238086

[12] A. Winter, A model for the fatigue of copper at low plastic strain amplitudes, Philosophical Magazine 30 (4) (1974) 719-738. doi:10.1080/14786437408207230

[13] P. J. Woods, Low-amplitude fatigue of copper and copper-5 at.\% aluminium single crystals, Philosophical Magazine 28 (1) (1973) 155-191. doi:10.1080/14786437308217440

[14] J. Man, P. Klapetek, O. Man, A. Weidner, K. Obrtlík, J. Polák, Extrusions and intrusions in fatigued metals. part 2. afm and ebsd study of the early growth of extrusions and intrusions in 3161 steel fatigued at room temperature, Philosophical Magazine 89 (16) (2009) 1337-1372. doi:10.1080/14786430902917624

[15] J. Man, K. Obrtlik, J. Polak, Extrusions and intrusions in fatigued metals. part 1. state of the art and history, Philosophical Magazine 89 (16) (2009) 1295-1336. doi:10.1080/14786430902917616

[16] L. M. Brown, Dislocation bowing and passing in persistent slip bands, Philosophical Magazine 86 (25-26) (2006) $4055-4068$. doi: 10.1080/14786430500501689

[17] S. Lavenstein, Y. Gu, D. Madisetti, J. A. El-Awady, The heterogeneity of persistent slip band nucleation and evolution in metals at the micrometer scale, Science 370 (6513). doi:10.1126/science.abb2690

[18] B. Gong, Z. Wang, Z. Wang, Cyclic deformation behavior and dislocation structures of [001] copper single crystalsi cyclic stress-strain response and surface feature, Acta materialia 45 (4) (1997) 1365-1377. doi:10.1016/S1359-6454(96) 00288-1

[19] A. S. Cheng, C. Laird, Mechanisms of fatigue hardening in copper single crystals: The effects of strain amplitude and orientation, Materials Science and Engineering 51 (1) (1981) 111-121. doi:10.1016/0025-5416(81)90112-9.

$610[20]$ A. Abel, Fatigue of copper single crystals at low constant plastic strain amplitudes, Materials Science and Engineering 36 (1) (1978) 117-124. doi:10.1016/0025-5416(78)90201-X.

[21] C. Feltner, C. Laird, Cyclic stress-strain response of fcc metals and alloysi phenomenological experiments, Acta metallurgica 15 (10) (1967) 1621-1632. doi:10.1016/0001-6160(67)90137-X

[22] C. Feltner, C. Laird, Factors influencing dislocation structures in fatigued metals, Transactions of the Metallurgical Society of AIME 242 (7) (1968) 1253.

[23] N. Clément, The order and disorder in materials, Aussois, France: Ecole dHiver.

[24] T. Steffens, C. Schwink, A. Korner, H. Karnthaler, Transmission electron microscopy study of the stacking-fault energy and dislocation structure in cumn alloys, Philosophical Magazine A 56 (2) (1987) 161-173. doi:10.1080/01418618708205159

[25] H. Mughrabi, R. Wang, Cyclic strain localization and fatigue crack initiation in persistent slip bands in face-centred cubic metals and single-phase alloys, in: Defects and Fracture, Springer, 1982, pp. 15-28. doi:10.1007/978-94-011-7520-3_2

[26] B. Devincre, Simulations dynamiques des dislocations a une echelle mesoscopique: une etude de la deformation plastique, Ph.D. thesis, Paris 11 (1993).

[27] M. Verdier, M. Fivel, I. Groma, Mesoscopic scale simulation of dislocation dynamics in fcc metals: Principles and applications, Modelling and Simulation in Materials Science and Engineering 6 (6) (1998) 755. doi:10.1088/0965-0393/6/6/007

[28] J. Yang, Y. Li, S. Li, C. Ma, G. Li, Simulation and observation of dislocation pattern evolution in the early stages of fatigue in a copper single crystal, Materials Science and Engineering: A 299 (1-2) (2001) 51-58. doi:10.1016/S0921-5093(00) 01415-5

[29] V. Deshpande, A. Needleman, E. Van der Giessen, Discrete dislocation plasticity modeling of short cracks in single crystals, Acta Materialia 51 (1) (2003) 1-15. doi:10.1016/S1359-6454(02)00401-9

$630[30]$ S. Brinckmann, E. Van der Giessen, A discrete dislocation dynamics study aiming at understanding fatigue crack initiation, Materials Science and Engineering: A 387 (2004) 461-464. doi:10.1016/j.msea.2004.01.142.

[31] C. Déprés, C. Robertson, M. Fivel, Low-strain fatigue in aisi 3161 steel surface grains: a three-dimensional discrete dislocation dynamics modelling of the early cycles i. dislocation microstructures and mechanical behaviour, Philosophical Magazine 84 (22) (2004) 2257-2275. doi:10.1080/14786430410001690051.

635 [32] C. Déprés, C. Robertson, M. C. Fivel, Low-strain fatigue in 3161 steel surface grains: a three dimension discrete dislocation dynamics modelling of the early cycles. part 2: Persistent slip markings and micro-crack nucleation, Philosophical Magazine 86 (1) (2006) 79-97. doi:10.1080/14786430500341250

[33] C. Déprés, C. Robertson, M. Fivel, 3d discrete dislocation dynamics investigations of fatigue crack initiation and propagation, Aerospace Lab Journal AL09 (01). doi:10.12762/2015.AL09-01

640 [34] C. Déprés, G. Prasad Reddy, C. Robertson, M. Fivel, An extensive 3d discrete dislocation dynamics investigation of stage-i fatigue crack propagation, Philosophical Magazine 94 (36) (2014) 4115-4137. doi:10.1080/14786435.2014.978830

[35] G. Prasad Reddy, C. Robertson, C. Déprés, M. Fivel, Effect of grain disorientation on early fatigue crack propagation in face-centred-cubic polycrystals: A three-dimensional dislocation dynamics investigation, Acta Materialia 61 (2013) 5300-5310. doi:10.1016/j.actamat.2013.05.021

[36] A. M. Hussein, J. A. El-Awady, Quantifying dislocation microstructure evolution and cyclic hardening in fatigued facecentered cubic single crystals, Journal of the Mechanics and Physics of Solids 91 (2016) 126-144. doi:10.1016/j.jmps. 2016.03.012

[37] A. M. Hussein, J. A. El-Awady, Surface roughness evolution during early stages of mechanical cyclic loading, International 
Journal of Fatigue 87 (2016) 339-350. doi:10.1016/j.ijfatigue.2016.02.022

[38] T. El Achkar, D. Weygand, Analysis of dislocation microstructure characteristics of surface grains under cyclic loading by discrete dislocation dynamics, Modelling and Simulation in Materials Science and Engineeringdoi:10.1088/1361-651X/ ab1b7c

[39] C. Erel, G. Po, N. Ghoniem, Dependence of hardening and saturation stress in persistent slip bands on strain amplitude during cyclic fatigue loading, Philosophical Magazine 97 (32) (2017) 2947-2970. doi:10.1080/14786435.2017.1361555

[40] M. Stricker, D. Weygand, P. Gumbsch, Irreversibility of dislocation motion under cyclic loading due to strain gradients, Scripta Materialia 129 (2017) 69-73. doi:10.1016/j.scriptamat.2016.10.029

[41] J. Senger, D. Weygand, O. Kraft, P. Gumbsch, Dislocation microstructure evolution in cyclically twisted microsamples: a discrete dislocation dynamics simulation, Modelling and Simulation in Materials Science and Engineering 19 (7) (2011) 074004. doi:10.1088/0965-0393/19/7/074004

42] C. Zhou, R. LeSar, Dislocation dynamics simulations of the bauschinger effect in metallic thin films, Computational Materials Science 54 (2012) 350-355. doi:10.1016/j.commatsci.2011.09.031

[43] S. S. Shishvan, L. Nicola, E. Van der Giessen, Bauschinger effect in unpassivated freestanding thin films, Journal of Applied Physics 107 (9) (2010) 093529. doi:10.1063/1.3407505

[44] L. Kubin, G. Canova, M. Condat, B. Devincre, V. Pontikis, Y. Bréchet, Dislocation licrostructures and plastic flow: a 3d simulation, Solid State Phenomena 23-24 (1992) 455-472. doi:10.4028/www.scientific.net/SSP.23-24.455

$45]$ J. V. Carstensen, Structural evolution and mechanisms of fatigue in polycrystalline brass, Ris $\varnothing$ National Laboratory, 1998.

[46] H. Mughrabi, Microscopic mechanisms of metal fatigue, in: Strength of metals and alloys, Elsevier, 1979, pp. 1615-1638. doi:10.1016/B978-1-4832-8412-5.50248-4

[47] Y. Li, C. Laird, Cyclic response and dislocation structures of aisi 3161 stainless steel. part 1: single crystals fatigued at intermediate strain amplitude, Materials Science and Engineering: A 186 (1-2) (1994) 65-86. doi:10.1016/0921-5093(94) 90306-9

[48] P. Li, S. Li, Z. Wang, Z. Zhang, Fundamental factors on formation mechanism of dislocation arrangements in cyclically deformed fcc single crystals, Progress in Materials Science 56 (3) (2011) 328-377. doi:10.1016/j.pmatsci.2010.12.001

[49] F. Kroupa, Dislocation dipoles and dislocation loops, Le Journal de Physique Colloques 27 (C3) (1966) C3-154. doi: 10.1051/jphyscol:1966320

[50] V. Gerold, H. Karnthaler, On the origin of planar slip in fcc alloys, Acta Metallurgica 37 (8) (1989) 2177-2183. doi: 10.1016/0001-6160(89) 90143-0

[51] Z. Basinski, A. Korbel, S. Basinski, The temperature dependence of the saturation stress and dislocation substructure in fatigued copper single crystals, Acta Metallurgica 28 (2) (1980) 191-207. doi:10.1016/0001-6160(80)90068-1

[52] M. Fivel, C. Déprés, An easy implementation of displacement calculations in 3d discrete dislocation dynamics codes, Philosophical Magazine 94 (28) (2014) 3206-3214. doi:10.1080/14786435.2014.949326

[53] M. Bao-Tong, C. Laird, Overview of fatigue behavior in copper single crystalsi. surface morphology and stage i crack initiation sites for tests at constant strain amplitude, Acta Metallurgica 37 (2) (1989) 325-336. doi:10.1016/0001-6160(89) 90217-4

54] K. Mecke, C. Blochwitz, Saturation dislocation structures in cyclically deformed nickel single crystals of different orientations, Crystal Research and Technology 17 (6) (1982) 743-758. doi:10.1002/crat.2170170610

[55] E.-W. Huang, R. I. Barabash, Y. Wang, B. Clausen, L. Li, P. K. Liaw, G. E. Ice, Y. Ren, H. Choo, L. M. Pike, et al., Plastic behavior of a nickel-based alloy under monotonic-tension and low-cycle-fatigue loading, International Journal of Plasticity 24 (8) (2008) 1440-1456. doi:10.1016/j.ijplas.2007.10.001

56] U. Essmann, H. Mughrabi, Annihilation of dislocations during tensile and cyclic deformation and limits of dislocation densities, Philosophical Magazine A 40 (6) (1979) 731-756. doi:10.1080/01418617908234871

[57] H. Mughrabi, Cyclic slip irreversibilities and the evolution of fatigue damage, Metallurgical and Materials Transactions B 40 (4) (2009) 431-453. doi:10.1007/s11663-009-9240-4

[58] D. Kuhlmann-Wilsdorf, Dislocation behavior in fatigue iii. properties of loop patchesdo they participate in fatigue cycling?, Materials Science and Engineering 39 (1) (1979) 127-139. doi:10.1016/0025-5416(79)90178-2

[59] M. Glazov, L. Llanes, C. Laird, Self-organized dislocation structures (sods) in fatigued metals, physica status solidi (a) 149 (1) (1995) 297-321. doi:10.1002/pssa.2211490121

[60] D. Kuhlmann-Wilsdorf, C. Laird, Dislocation behavior in fatigue, Materials Science and Engineering 27 (2) (1977) $137-156$. doi:10.1016/0025-5416(77)90166-5

700 [61] Z. Wang, Cyclic deformation response of planar-slip materials and a new criterion for the wavy-to-planar-slip transition, Philosophical Magazine 84 (3-5) (2004) 351-379. doi:10.1080/14786430310001639824

[62] S. I. Hong, C. Laird, Mechanisms of slip mode modification in fcc solid solutions, Acta metallurgica et materialia 38 (8) (1990) 1581-1594. doi:10.1016/0956-7151(90)90126-2 